\title{
NUMERICAL APPROACHES TO RATE-INDEPENDENT PROCESSES AND APPLICATIONS IN INELASTICITY*
}

\author{
Alexander Mielke ${ }^{1,2}$ And Tomáš RoubíČEK ${ }^{3,4}$
}

\begin{abstract}
A conceptual numerical strategy for rate-independent processes in the energetic formulation is proposed and its convergence is proved under various rather mild data qualifications. The novelty is that we obtain convergence of subsequences of space-time discretizations even in case where the limit problem does not have a unique solution and we need no additional assumptions on higher regularity of the limit solution. The variety of general perspectives thus obtained is illustrated on several specific examples: plasticity with isotropic hardening, damage, debonding, magnetostriction, and two models of martensitic transformation in shape-memory alloys.
\end{abstract}

Mathematics Subject Classification. 35K85, 49J40, 49S05, 65J15, 65M12, 65Z05, 74C05, 74F15, 74H15, 74N10, 74R05, 74S05.

Received November 13, 2006. Revised January 31, 2008.

Published online April 8, 2009.

\section{INTRODUCTION}

Fully rate independent models for processes describing material models occur as limits when the loading rate slows down to 0 . This makes the model simpler by omitting all effects due to interior relaxation processes. However, the resulting rate-independent mathematical models are somehow degenerate. In particular, in many cases solutions for a given initial datum are no longer unique and may have jumps in time.

Nevertheless such models, being a subclass of the generalized standard materials [16,21], are widely used in engineering as they provide good mesoscopic descriptions of hysteretic response during isothermal evolution processes. Mathematical analysis of such processes, based on the notion of energetic solutions introduced in $[41,44]$, has been intensively scrutinized and developed in particular in $[30,34,37,39,40,42,43,56]$. However,

\footnotetext{
Keywords and phrases. Rate-independent evolution, energetic solution, approximation, plasticity, damage, debonding, magnetostriction, martensitic transformation.

* The second author acknowledges the hospitality of WIAS Berlin, where the majority of this research has been carried out, supported through the Alexander von Humboldt Foundation. Partial support is acknowledged from the DFG-Research Center "MATHeON" (C18), the grants A 1075402 and IAA 100750802 (GA AV CR), LC 06052, MSM 21620839, and 1M06031

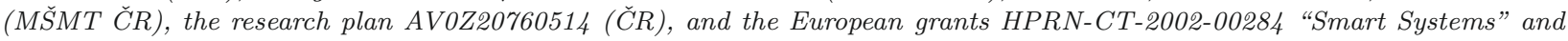
MRTN-CT-2004-505226 "Multi-scale modelling and characterisation for phase transformations in advanced materials".

1 Weierstraß-Institut für Angewandte Analysis und Stochastik, Mohrenstr. 39, 10117 Berlin, Germany.

2 Institut für Mathematik, Humboldt Universität zu Berlin, Rudower Chaussee 25, 12489 Berlin, Germany.

3 Mathematical Institute, Charles University, Sokolovská 83, 18675 Praha 8, Czech Republic. roubicek@karlin.mff.cuni.cz

${ }^{4}$ Institute of Thermomechanics of the ASCR, Dolejškova 5, 18200 Praha 8, Czech Republic.
} 
except for some very particular cases $[7,18,22,23,31,57]$, there has been no numerical analysis developed for such processes so far.

This paper provides a universally-applicable numerical scheme in the context of rate-independent processes and its convergence analysis. In particular, it is suited to cases where we do not have uniqueness of solutions and where solutions may even have jumps in time. In such a situation we cannot hope to establish convergence rates nor convergence of the whole approximation sequence. We rather obtain that subsequences exist that converge to solutions, which may be considered as weak version of "stability" of our scheme. In fact, any limit that is obtained from a converging subsequence of approximations solves the rate-independent system, which may be considered as weak form of "consistency".

To present the main ideas and results we expose first a simplified case. Consider a Banach space $Q$ serving as a state space. The system is described by an energy functional $E: Q \rightarrow \mathbb{R}$ and a dissipation potential $R: Q \rightarrow[0, \infty]$, the latter being convex and homogeneous of degree 1. Imposing a time-dependent loading $f:[0, T] \rightarrow Q^{*}$, the classical evolution law is the following differential inclusion, which is a (generalized) force balance:

$$
f(t) \in \partial R\left(\frac{\mathrm{d} q}{\mathrm{~d} t}(t)\right)+E^{\prime}(q(t)) \quad \text { a.e. on }[0, T]
$$

where $\partial$ stands for the subdifferential and $E^{\prime}$ is the Gâteaux derivative of $E$. Rate independence means the fact that $q$ solves the problem for the loading $f$ if and only if for all $\lambda>0$ the function $q_{\lambda}: t \mapsto q(\lambda t)$ solves (1.1) with loading $f_{\lambda}: t \mapsto f(\lambda t)$. This property easily follows from $\partial R(v)=\partial R(\lambda v)$, which implies that (1.1) is a nonsmooth problem as $v \mapsto \partial R(v)$ is not continuous.

However, for general $E$ that is nonsmooth or nonconvex, we need a weak form of (1.1), which allows for solutions $q$ having jumps with respect to time $t$ and for energies $E$ being nonsmooth or nonconvex. In the rateindependent setting such a weak form is given via the energetic formulation (S) and (E). A process $q:[0, T] \rightarrow Q$ is called an energetic solution if, for all $t \in[0, T]$, we have

$$
\begin{array}{lll}
\text { stability (S): } & \forall \tilde{q} \in Q: \quad E(q(t))-\langle f(t), q(t)\rangle \leq E(q(0))-\langle f(0), q(0)\rangle+R(\tilde{q}-q(t)), \\
\text { energy balance (E): } & E(q(t))-\langle f(t), q(t)\rangle+\int_{0}^{t} R(\mathrm{~d} q)=E(q(0))-\langle f(0), q(0)\rangle-\int_{0}^{t}\left\langle\frac{\mathrm{d} f}{\mathrm{~d} t}(s), q(s)\right\rangle \mathrm{d} s
\end{array}
$$

where $\langle\cdot, \cdot\rangle$ is the duality pairing between $Q^{*}$ and $Q$. As observed in [42], every differentiable solution $q$ of (1.2) solves (1.1). To see this one exploits that $R$ is homogeneous of degree 1, giving (i) $\partial R(v) \subset \partial R(0)$ for all $v$ and (ii) $\eta \in \partial R(v)$ if and only if $\eta \in \partial R(0)$ and $\langle\eta, v\rangle=R(v)$. Now (S) implies $f \in \partial R(0)+E^{\prime}(q)$, and differentiating (E) with respect to time gives $\left\langle E^{\prime}(q), \frac{\mathrm{d} q}{\mathrm{~d} t}\right\rangle+R\left(\frac{\mathrm{d} q}{\mathrm{~d} t}\right)=0$. Together with (i) and (ii) we obtain (1.1). The opposite inclusion that (1.1) implies (1.2) holds if $E$ is uniformly convex, see [42].

The importance of the energetic formulation (1.2) is that it is derivative free and thus allows for more general functionals and solutions. In particular approximation results are easier as we will not need higher regularity of solutions. A more general setting on topological spaces is introduced in Section 2.1, as the main ideas introduced in this work are not restricted to Banach spaces and can be applied to a wider class of problems than exploited in this paper. In particular, they are adjusted to treat geometrically nonlinear problems like finite-strain elastoplasticity [35,36,38], gradient-Young measures as considered in [31], and for brittle fracture and crack propagation, see [7].

First we replace the full space $Q$ by a family $\left(Q_{h}\right)_{h>0}$ of finite-dimensional subspaces that exhausts $Q$ in the sense that each $q \in Q$ is the limit of a sequence $\left(q_{h}\right)_{h>0}$ with $q_{h} \in Q_{h}$. Second we introduce a partition $0=t_{\tau}^{0}<t_{\tau}^{1}<\ldots<t_{\tau}^{k_{\tau}}=T$ of the time interval $[0, T]$ with fineness $\tau:=\max \left\{t_{\tau}^{j}-t_{\tau}^{j-1} ; j=1, \ldots, k_{\tau}\right\}$. The algorithm we propose is the following incremental problem, combining the fully implicit Euler method with the 
finite-dimensional discretization: given $\left[q_{0}\right]_{h} \in Q_{h}$, find $q_{h, \tau}^{1}, \ldots, q_{\tau, h}^{k_{\tau}} \in Q_{h}$ satisfying

$$
\left.\begin{array}{ll}
\text { Minimize } & E\left(q_{h}\right)-\left\langle f\left(t_{\tau}^{k}\right), q_{h}\right\rangle+R\left(q_{h}-q_{\tau, h}^{k-1}\right) \\
\text { subject to } & q_{h} \in Q_{h} .
\end{array}\right\}
$$

Again it is the rate-independence that makes the time-incremental problem especially simple. We have to minimize the sum of the total energy and the dissipated energy in the given time step, but the length of the time step does not appear. However the evolutionary character is inherited by the occurrence of $q_{\tau, h}^{k-1}$. The intrinsic link between this time-incremental minimization problem and the energetic formulations is manifested in Proposition 2.3, where we prove that the discrete solutions satisfy a discrete version of the stability condition (S) and upper and lower a priori energy bounds replacing (E), see (1.2).

The convergence result in Theorem 2.6 for the simultaneous passage to the limit $\tau, h \rightarrow 0$ relies on an abstract joint recovery condition (see (2.19)), which was first established in [45] for general $\Gamma$-convergence results. If $\bar{q}_{\tau, h}:[0, T] \rightarrow Q_{h} \subset Q$ denote the piecewise constant interpolants of the solutions of (1.3), then there exists a subsequence $\left(\tau_{n}, h_{n}\right) \rightarrow(0,0)$ and a solution $q:[0, T] \rightarrow Q$ of $(1.2)$ such that $q_{n}=\bar{q}_{\tau_{n}, h_{n}}$ satisfies the following convergence properties for all $t \in[0, T]$ :

$$
q_{n}(t) \rightarrow q(t), \quad E\left(q_{n}(t)\right) \rightarrow E(q(t)), \quad \int_{0}^{t} R\left(\mathrm{~d} q_{n}\right) \rightarrow \int_{0}^{t} R(\mathrm{~d} q),
$$

see Theorem 2.6 for the exact statement. In fact, the result is a consequence of the abstract $\Gamma$-convergence theory developed in [45]. The point is that the concept of $\Gamma$-convergence is sufficiently general to deal with approximation of functionals via finite-dimensional subspaces.

In summary, the abstract results can be reduced to two independent principles that are close to the well-known principles of stability and consistency in numerical analysis. First, the discrete energetic a priori estimates show that the solutions $\bar{q}_{\tau, h}$ are bounded independently of $\tau$ and $h$, which is a weak form of stability. Second, the $\Gamma$-convergence theory implies that any limit point for $(\tau, h) \rightarrow(0,0)$ of the approximations $\bar{q}_{\tau, h}$ is an energetic solution, which is weak form of consistency that is commonly used for problems where we have to expect multiple solutions.

In Section 3, the generality is reduced to problems posed on Banach spaces, which in turn allows for various specific constructions directly applicable in concrete situations. For instance, the joint recovery condition is satisfied, if $E$ is weakly lower semicontinuous and strongly continuous while $R$ is weakly continuous. Moreover, while the above version is restricted to the conforming case $Q_{h} \subset Q$, we now can also allow for a constraint mapping $\Xi: Q \rightarrow X$, which will be added to $E$ in the form of the penalty term $\frac{1}{\varepsilon}\|\Xi(\cdot)\|_{X}^{\alpha}$. In such a way nonconforming methods can be included as well. For the augmented functional $E_{\varepsilon}:=E+\frac{1}{\varepsilon}\|\Xi(\cdot)\|_{X}^{\alpha}$ we obtain the discretized solutions $\bar{q}_{\tau, h, \varepsilon}$. The abstract convergence result presented in Theorem 2.6 is already fitted to this situation and convergence is obtained under a possible additional restriction $0<h_{n}<H\left(\varepsilon_{n}\right)$.

This is illustrated in Section 4 on various examples from continuum mechanics of deformable bodies, namely plasticity with hardening, two models of martensitic transformation, damage, debonding, and magnetostriction. In particular, it accompanies a large variety of existing models by conceptual finite-element discretizations supported by rigorous analysis as far as convergence concerns, and in some cases offers new results or improves known results as far as mere existence of solutions concerns.

\section{The ABStract SETting AND APPROXIMATION RESUlT}

In this section we first present the abstract energetic formulation for rate-independent systems. This formulation encompasses a wide variety of engineering models for materials with internal variables or/and activated processes on the boundary. The advantage of the energetic formulation is that it is derivative free and thus applies in situations where the constitutive laws are not continuous and where solutions may have jumps. 
Moreover, the approach is based in incremental minimization and thus methods for the calculus of variations are applicable. Second we present a simplified version of the $\Gamma$-convergence theory developed in [45]. The basis of this work is the general observation that numerical approximation for the energetic formulation of rate-independent processes can be interpreted as a special case of $\Gamma$-convergence.

We emphasize that our formulation is kept in the rather abstract form of rate-independent systems $(\mathcal{Q}, \mathcal{E}, \mathcal{D})$, where $\mathcal{Q}$ is the state space, $\mathcal{D}$ the dissipation distance (see below), and $\mathcal{E}$ a general Gibbs-type stored energy $\mathcal{E}:[0, T] \times \mathcal{Q} \rightarrow \mathbb{R} \cup\{+\infty\}$, which may be more general than the form $\mathcal{E}(t, q)=E(q)-\langle f(t), q\rangle$ used in Section 1 . Ultimately this general form may not be needed for the applications treated later in Section 4; however, adapting the theory to a Banach-space setting and/or the mentioned special form $\mathcal{E}(t, \cdot)=E+f(t)$ would not simplify the presentation, but would make it even less lucid, notationally less elegant, and destroy the comparability with the developments in [45], which are our basis. Moreover, the general form for $\mathcal{E}$ is needed for treating timevarying Dirichlet boundary conditions $q_{\mathrm{D}}:[0, T] \rightarrow Q$ that give rise to the form $\mathcal{E}(t, q)=E\left(q-q_{\mathrm{D}}(t)\right)-\langle f(t), q\rangle$, see e.g. in $[14,40]$. Finally, we mention that the abstract theory applies also in cases where there is no linear (Banach-space) structure, namely for the evolution of gradient-Young measures [31], for brittle fracture and crack propagation [7], and for finite-strain elastoplasticity [35,36,38], although we will not discuss these applications here.

\subsection{The energetic formulation}

We consider a state space $\mathcal{Q}$ (independent of time) as a topological space. Typically, but not necessarily it is subset of a Banach space. We will distinguish between a "non-dissipative" component $u \in \mathcal{U}$ and a "dissipative" component $z \in \mathcal{Z}$ of the state $q=(u, z) \in \mathcal{Q}:=\mathcal{U} \times \mathcal{Z}$.

For a fixed time horizon $T>0$, we consider a Gibbs-type stored energy $\mathcal{E}:[0, T] \times \mathcal{Q} \rightarrow \mathbb{R} \cup\{+\infty\}$. The further ingredient is a time-independent dissipation distance $\mathcal{D}: \mathcal{Z} \times \mathcal{Z} \rightarrow[0,+\infty]$ which will later determine the dissipated energy and which is assumed to satisfy

$$
\forall z_{1}, z_{2}, z_{3} \in \mathcal{Z}: \quad\left(\mathcal{D}\left(z_{1}, z_{2}\right)=0 \Leftrightarrow z_{1}=z_{2}\right) \quad \& \quad \mathcal{D}\left(z_{1}, z_{3}\right) \leq \mathcal{D}\left(z_{1}, z_{2}\right)+\mathcal{D}\left(z_{2}, z_{3}\right) .
$$

Note that we allow for an unsymmetric distance $\mathcal{D}$, i.e., $\mathcal{D}\left(z_{1}, z_{2}\right) \neq \mathcal{D}\left(z_{2}, z_{1}\right)$ is allowed, and strictly spoken $\mathcal{D}$ is only a quasi-metric. Let us agree to write occasionally $\mathcal{D}\left(q_{1}, q_{2}\right)$ with the meaning $\mathcal{D}\left(z_{1}, z_{2}\right)$ for $q_{1}=\left(u_{1}, z_{1}\right)$ and $q_{2}=\left(u_{2}, z_{2}\right)$.

In case of $Q$ having a linear structure, $\mathcal{D}\left(z_{1}, z_{2}\right):=R\left(z_{2}-z_{1}\right)$ (as in Sect. 3 below) and convexity of both, $\mathcal{E}(t, \cdot)$ and $R$, we want to address an evolution of $q=q(t)$ governed by the doubly nonlinear inclusion

$$
\partial R\left(\frac{\partial q}{\partial t}\right)+\partial_{q} \mathcal{E}(t, q) \ni 0
$$

where " $\partial$ " denotes the subdifferential and $\partial_{q} \mathcal{E}(t, q)$ the "partial" subdifferential of $\mathcal{E}(t, \cdot)$. Under some additional qualification, it is equivalent (see [37,42]) to the energetic formulation based on Definition 2.1 below which, however, works under much weaker data qualification where (2.2) loses any sense. In fact, this definition is based on a global-minimization hypothesis competing with the maximum-dissipation principle (or rather Levitas' realizability principle [32]). In mathematical terms, we are led to stability

$$
\forall \tilde{q} \in \mathcal{Q}: \quad \mathcal{E}(t, q(t)) \leq \mathcal{E}(t, \tilde{q})+\mathcal{D}(q(t), \tilde{q}),
$$

and energy equality

$$
\begin{aligned}
& \mathcal{E}(t, q(t))+\operatorname{Var}_{\mathcal{D}}(q ; s, t)=\mathcal{E}(s, q(s))+\int_{s}^{t} \mathcal{P}(r, q(r)) \mathrm{d} r, \quad \text { where } \\
& \mathcal{P}(t, q):=\frac{\partial}{\partial t} \mathcal{E}(t, q) \quad \text { and } \quad \operatorname{Var}_{\mathcal{D}}(q ; s, t):=\sup \sum_{i=1}^{j} \mathcal{D}\left(q\left(t_{i-1}\right), q\left(t_{i}\right)\right)
\end{aligned}
$$


with the supremum taken over all $j \in \mathbb{N}$ and over all partitions of $[s, t]$ in the form $s=t_{0}<t_{1}<\ldots<t_{j-1}<$ $t_{j}=t$. The particular terms in (2.4a) represent the stored energy at time $t$, the energy dissipated by changes of the internal variable during the time interval $[s, t]$, the stored energy at the initial time $s$, and the work done by external loadings during the time interval $[s, t]$; and $\mathcal{P}$ is then the power.

Definition 2.1. The process $q:[0, T] \rightarrow \mathcal{Q}$ is called an energetic solution to the initial-value problem given by $\left(\mathcal{Q}, \mathcal{E}, \mathcal{D}, q_{0}\right)$ if

(i) it is stable in the sense that (2.3) holds for all $t \in[0, T]$;

(ii) the energy balance (2.4a) holds for all $0 \leq s<t \leq T$; in particular $t \mapsto \mathcal{P}(t, q(t))$ is in $L^{1}(0, T)$; and

(iii) the initial condition $q(0)=q_{0}$ holds.

For the analysis of the rate-independent problems, it is convenient to introduce the sets of stable states $\mathcal{S}(t)$ for any $t \in[0, T]$ by putting

$$
\mathcal{S}(t):=\{q \in \mathcal{Q} ; \quad \mathcal{E}(t, q)<+\infty \quad \& \quad \forall \tilde{q} \in \mathcal{Q}: \quad \mathcal{E}(t, q) \leq \mathcal{E}(t, \tilde{q})+\mathcal{D}(q, \tilde{q})\}
$$

This allows us to recast the stability condition (i) in Definition 2.1 in the form $q(t) \in \mathcal{S}(t)$ for all $t \in[0, T]$. Yet, more importantly, we may address closeness properties of $\mathcal{S}(t)$.

In Section 3, we will specialize this setting by introducing an additional linear structure, i.e. $\mathcal{Q}$ will be (a subset of) a Banach space equipped with the weak or the norm topology. This will allow us to make the abstract properties more specific.

\subsection{The abstract assumptions}

For our abstract approximation we consider three positive parameters $\tau, h$, and $\varepsilon$. Here $\tau>0$ represents the fineness of a time discretization by a partition (not necessarily equidistant) of the time interval $[0, T]$. The parameter $h>0$ denotes a discretization of the state space $\mathcal{Q}$ by a subset $\mathcal{Q}_{h}$ again having the structure $\mathcal{Q}_{h}:=\mathcal{U}_{h} \times \mathcal{Z}_{h}$. We assume that each $\mathcal{Q}_{h}$ is closed and the family $\left(\mathcal{Q}_{h}\right)_{h>0}$ is dense in the sense that for each $q \in \mathcal{Q}$ there exist $q_{h} \in \mathcal{Q}_{h}$ such $q_{h} \rightarrow q$. The small parameter $\varepsilon>0$ is used for a possible approximation of the functionals $\mathcal{E}$ and $\mathcal{D}$ to be implemented more easily when restricted on $\mathcal{Q}_{h}$ (see also Rem. 2.8 below) or just to guarantee the convergence in some more complicated cases. Typically, a penalization of some constraints may be involved by this way, $c f$. Section 4 . These last approximations lead to $\mathcal{E}_{\varepsilon}:[0, T] \times \mathcal{Q} \rightarrow \mathbb{R} \cup\{+\infty\}$ and $\mathcal{D}_{\varepsilon}: \mathcal{Z} \times \mathcal{Z} \rightarrow \mathbb{R} \cup\{+\infty\}$.

Using the indicator function $\delta_{\mathcal{Q}_{h}}: \mathcal{Q} \rightarrow\{0,+\infty\}$, i.e. $\delta_{\mathcal{Q}_{h}}=0$ on $\mathcal{Q}_{h}$ and $\delta_{\mathcal{Q}_{h}}=+\infty$ on $\mathcal{Q} \backslash \mathcal{Q}_{h}$, it will occasionally be convenient to introduce the restriction to $\mathcal{Q}_{h}$ also by replacing $\mathcal{E}_{\varepsilon}$ and $\mathcal{D}_{\varepsilon}$ respectively by

$$
\mathcal{E}_{\varepsilon, h}=\mathcal{E}_{\varepsilon}+\delta_{\mathcal{Q}_{h}} \quad \text { and } \quad \mathcal{D}_{\varepsilon, h}:(q, \tilde{q}) \mapsto \mathcal{D}_{\varepsilon}(q, \tilde{q})+\delta_{\mathcal{Q}_{h}}(q)+\delta_{\mathcal{Q}_{h}}(\tilde{q}) .
$$

We now collect a few basic assumptions. We include the limit case $(\mathcal{Q}, \mathcal{E}, \mathcal{D})$ by setting $\mathcal{Q}_{0}=\mathcal{Q}, \mathcal{E}_{0}=\mathcal{E}$, and $\mathcal{D}_{0}=\mathcal{D}$ and by including $\varepsilon=0$ and $h=0$ if desired. Like in (2.1), we assume that each $\mathcal{D}_{\varepsilon}$, $\varepsilon \geq 0$, satisfies a quasi-metric property:

$$
\forall z_{1}, z_{2}, z_{3} \in \mathcal{Z}:\left(\mathcal{D}_{\varepsilon}\left(z_{1}, z_{2}\right)=0 \Leftrightarrow z_{1}=z_{2}\right) \quad \& \quad \mathcal{D}_{\varepsilon}\left(z_{1}, z_{3}\right) \leq \mathcal{D}_{\varepsilon}\left(z_{1}, z_{2}\right)+\mathcal{D}_{\varepsilon}\left(z_{2}, z_{3}\right) .
$$

For proving existence results we will need the following lower semicontinuity and compactness results:

$$
\forall \varepsilon, h \geq 0: \quad \mathcal{D}_{\varepsilon}: \mathcal{Q}_{h} \times \mathcal{Q}_{h} \rightarrow \mathbb{R}_{\infty} \text { are lower semicontinuous. }
$$

Next we need a "$\Gamma$-liminf estimate" for the family $\left(\mathcal{D}_{\varepsilon}\right)_{\varepsilon>0}$ on $\left(\mathcal{Q}_{h} \times \mathcal{Q}_{h}\right)_{h>0}$ in the limit $\varepsilon, h \rightarrow 0$ :

$$
\left.\begin{array}{l}
z \in \mathcal{Z}, \quad z_{h, \varepsilon} \in \mathcal{Z}_{h}, \quad z=\lim _{(h, \varepsilon) \rightarrow(0,0)} z_{h, \varepsilon} \\
\tilde{z} \in \mathcal{Z}, \quad \tilde{z}_{h, \varepsilon} \in \mathcal{Z}_{h}, \quad \tilde{z}=\lim _{(h, \varepsilon) \rightarrow(0,0)} \tilde{z}_{h, \varepsilon}
\end{array}\right\} \Rightarrow \mathcal{D}(z, \tilde{z}) \leq \liminf _{(h, \varepsilon) \rightarrow(0,0)} \mathcal{D}_{\varepsilon}\left(z_{h, \varepsilon}, \tilde{z}_{h, \varepsilon}\right) .
$$


Note that our conditions (2.7) and (2.9) imply $z_{h, \varepsilon} \rightarrow z$ whenever $\min \left(\mathcal{D}\left(z_{h, \varepsilon}, z\right), \mathcal{D}\left(z, z_{h, \varepsilon}\right)\right) \rightarrow 0$ and $\left(z_{h, \varepsilon}\right)_{h, \varepsilon>0}$ is compact, i.e. the "positivity condition" [45], Assumption (2.4).

The conditions for the energy functionals are as follows:

$$
\forall \varepsilon, h \geq 0 \quad \forall t \in[0, T] \quad \forall a \in \mathbb{R} \text { : the sublevels }\left\{q \in \mathcal{Q}_{h} ; \mathcal{E}_{\varepsilon}(t, q) \leq a\right\} \text { are sequentially compact in } \mathcal{Q} .
$$

To pass to the limit will need a uniform inf-compactness of the collection $\left(\mathcal{E}_{\varepsilon, h}\right)_{\varepsilon, h>0}$ :

$$
\begin{aligned}
& \forall a \in \mathbb{R} \quad \forall\left(t_{\varepsilon, h}, q_{h, \varepsilon}\right) \in[0, T] \times \mathcal{Q}_{h}, \quad \text { with } \quad \mathcal{E}_{\varepsilon}\left(t_{\varepsilon, h}, q_{h, \varepsilon}\right) \leq a \\
& \exists q \in \mathcal{Q} \quad \exists \text { subsequence }\left\{q_{h_{n}, \varepsilon_{n}}\right\}_{n \in \mathbb{N}}: \quad q=\lim _{n \rightarrow \infty} q_{h_{n}, \varepsilon_{n}} .
\end{aligned}
$$

Like for $\mathcal{D}_{\varepsilon}$ we also need a " $\Gamma$-liminf estimate" for the family $\left(\mathcal{E}_{\varepsilon, h}\right)_{\varepsilon, h>0}$ :

$$
\forall(t, q) \in[0, T] \times \mathcal{Q} \quad \forall q_{h, \varepsilon} \in \mathcal{Q}_{h} \text { with } q=\lim _{(h, \varepsilon) \rightarrow(0,0)} q_{h, \varepsilon}: \quad \mathcal{E}(t, q) \leq \liminf _{(h, \varepsilon, \theta) \rightarrow(0,0, t)} \mathcal{E}_{\varepsilon}\left(\theta, q_{h, \varepsilon}\right) .
$$

Note that (2.9) and (2.12) are only "lower" $\Gamma$-liminf estimates for $\left(\mathcal{D}_{\varepsilon, h}\right)_{\varepsilon, h>0}$ and $\left(\mathcal{E}_{\varepsilon, h}\right)_{\varepsilon, h>0}$. The corresponding upper estimates are consequences of the central condition (2.19) postulating existence of joint recovery sequences.

So far all conditions above relate to static concepts. The next three conditions relate to the time dependence, which involves the power of external forces $\mathcal{P}_{\varepsilon}(t, q)=\frac{\partial}{\partial t} \mathcal{E}_{\varepsilon}(t, q)$. The first assumption provides a uniform energetic control of the power $\mathcal{P}_{\varepsilon}$, viz.,

$$
\begin{aligned}
\exists c_{0}, c_{1} \in \mathbb{R} \quad \forall \varepsilon>0 \quad \forall q \in \mathcal{Q}_{h} \text { with } \mathcal{E}_{\varepsilon}(0, q)<+\infty: & \mathcal{E}_{\varepsilon}(\cdot, q) \in C^{1}([0, T]) \quad \text { and } \\
& \forall t \in[0, T]:\left|\mathcal{P}_{\varepsilon}(t, q)\right| \leq c_{1}\left(\mathcal{E}_{\varepsilon}(t, q)+c_{0}\right) .
\end{aligned}
$$

Using a Gronwall estimate we immediately obtain the growth restrictions

$$
\forall s, t \in[0, T]: \mathcal{E}_{\varepsilon}(s, q)+c_{0} \leq \mathrm{e}^{c_{1}|t-s|}\left(\mathcal{E}_{\varepsilon}(t, q)+c_{0}\right) .
$$

The second assumption is a conditioned (with respect to sublevels of $\mathcal{E}$ ) equi- (with respect to $q$ ) uniform (with respect to $t$ ) continuity of $\mathcal{P}(\cdot, q)$ :

$$
\forall a \in \mathbb{R} \quad \forall \sigma>0 \quad \exists \delta>0 \quad \forall s, t \in[0, T] \quad \forall q \in \mathcal{Q} \text { : if } \mathcal{E}(0, q) \leq a \text { and }|t-s|<\delta \text {, then }|\mathcal{P}(s, q)-\mathcal{P}(t, q)|<\sigma .
$$

The third assumption on $\mathcal{P}_{\varepsilon, h}$ concerns the convergence of $\mathcal{P}_{\varepsilon, h}$ for $\varepsilon, h \rightarrow 0$. It is a "continuous convergence" but conditioned by the fact that the considered arguments are in the associated sets of stable states

$$
\mathcal{S}_{\varepsilon, h}(t):=\left\{q \in \mathcal{Q}_{h} ; \quad \mathcal{E}_{\varepsilon}(t, q)<+\infty \quad \& \quad \forall \tilde{q} \in \mathcal{Q}_{h}: \quad \mathcal{E}_{\varepsilon}(t, q) \leq \mathcal{E}_{\varepsilon}(t, \tilde{q})+\mathcal{D}_{\varepsilon}(q, \tilde{q})\right\}
$$

and that the energies are bounded:

$$
\begin{array}{r}
\text { If }\left(\varepsilon_{n}, h_{n}, t_{n}\right) \rightarrow(0,0, t), \quad q_{n} \in \mathcal{S}_{\varepsilon_{n}, h_{n}}\left(t_{n}\right), q_{n} \rightarrow q, \text { and } \\
\sup _{n \in \mathbb{N}} \mathcal{E}_{\varepsilon_{n}, h_{n}}\left(t_{n}, q_{n}\right)<+\infty, \text { then } \lim _{n \rightarrow \infty} \mathcal{P}_{\varepsilon_{n}}\left(t_{n}, q_{n}\right)=\mathcal{P}(t, q) .
\end{array}
$$

Recall that $\mathcal{D}_{\varepsilon}$ and $\mathcal{D}$ only depend on the $z$-component of $q=(u, z)$ and we have agreed to write occasionally, as e.g. in $(2.16), \mathcal{D}_{\varepsilon}(q, \tilde{q})$ in the meaning of $\mathcal{D}_{\varepsilon}(z, \tilde{z})$.

All the assumptions of the previous subsection are either on the family $\left(\mathcal{D}_{\varepsilon, h}\right)_{\varepsilon, h>0}$ or on the family $\left(\mathcal{E}_{\varepsilon, h}\right)_{\varepsilon, h>0}$. The final condition links the behavior of these two families and thus provide the upper $\Gamma$-limit estimates which are needed to complement the lower $\Gamma$-limit estimate for $\mathcal{D}$ in (2.9) and for $\mathcal{E}$ in (2.12). Sometimes, in particular 
when some holonomic-type constraints are involved in $\mathcal{E}$, it occurs that a convergence criterion of the type $h \leq H(\varepsilon)$, for some $H: \mathbb{R}^{+} \rightarrow \mathbb{R}^{+}$monotone and satisfying $H(\varepsilon) \rightarrow 0$ for $\varepsilon \rightarrow 0$, is needed.

Hence, our final condition in the interaction of $\mathcal{E}_{\varepsilon, h}$ and $\mathcal{D}_{\varepsilon, h}$ is the following conditioned upper semicontinuity of the sets of stable states:

$$
\begin{aligned}
& \forall q, \tilde{q} \in \mathcal{Q} \quad \forall t_{n} \in[0, T] \text { with } t_{n} \rightarrow t \forall \varepsilon_{n}, h_{n} \rightarrow 0 \text { with } h_{n} \leq H\left(\varepsilon_{n}\right) \\
& \forall q_{n} \in \mathcal{S}_{\varepsilon_{n}, h_{n}}\left(t_{n}\right) \text { with } q_{n} \rightarrow q \text { and } \sup _{n \in \mathbb{N}} \mathcal{E}_{\varepsilon_{n}, h_{n}}\left(t_{n}, q_{n}\right)<+\infty: \quad q \in \mathcal{S}(t) .
\end{aligned}
$$

The above assertion says, in other words, that the graph of the set-valued mapping $\mathcal{S}:[0, T] \rightrightarrows \mathcal{Q}$ contains Kuratowski's limes superior of the graphs of $\mathcal{S}_{\varepsilon, h}:[0, T] \rightrightarrows \mathcal{Q}_{h}$ at least if restricted to states with bounded energy as in (2.11) and if $h \leq H(\varepsilon)$ is taken into account. This upper semicontinuity result establishes a certain stability of sets of stable states that is crucial for the convergence analysis.

The following result gives a necessary condition for the above property (2.18). For various other conditions we refer to [45], Section 2. The result is based on the existence of a "joint recovery sequence" under suitable qualifications:

$$
\begin{aligned}
& \forall q, \tilde{q} \in \mathcal{Q} \quad \forall t_{n} \in[0, T] \text { with } t_{n} \rightarrow t \forall \varepsilon_{n}, h_{n} \rightarrow 0+\text { with } h_{n} \leq H\left(\varepsilon_{n}\right) \\
& \forall q_{n} \in \mathcal{S}_{\varepsilon_{n}, h_{n}}\left(t_{n}\right) \text { with } q_{n} \rightarrow q \text { and } \sup _{n \in \mathbb{N}} \mathcal{E}_{\varepsilon_{n}, h_{n}}\left(t_{n}, q_{n}\right)<+\infty \\
& \exists \tilde{q}_{n} \in \mathcal{Q}_{h_{n}} \text { with } \tilde{q}_{n} \rightarrow \tilde{q}: \\
& \limsup _{n \rightarrow \infty}\left(\mathcal{E}_{\mathcal{E}_{n}, h_{n}}\left(t_{n}, \tilde{q}_{n}\right)+\mathcal{D}_{\varepsilon_{n}, h_{n}}\left(q_{n}, \tilde{q}_{n}\right)-\mathcal{E}_{\varepsilon_{n}, h_{n}}\left(t_{n}, q_{n}\right)\right) \leq \mathcal{E}(t, \tilde{q})+\mathcal{D}(q, \tilde{q})-\mathcal{E}(t, q) .
\end{aligned}
$$

Lemma 2.2. Conditions (2.12) and (2.19) imply (2.18).

Proof. By (2.12) we have $\mathcal{E}(t, q) \leq \liminf _{n \rightarrow \infty} \mathcal{E}_{\varepsilon_{n}, h_{n}}\left(t_{n}, q_{n}\right) \leq \sup _{n \in \mathbb{N}} \mathcal{E}_{\varepsilon_{n}, h_{n}}\left(t_{n}, q_{n}\right)<\infty$, where the last inequality is assumed in (2.19). Next, for $\tilde{q} \in \mathcal{Q}$ arbitrary, choose $\tilde{q}_{n} \in \mathcal{Q}_{h_{n}}$ as in (2.19). By Definition (2.16), $q_{n} \in \mathcal{S}_{\varepsilon_{n}, h_{n}}\left(t_{n}\right)$ says that $\mathcal{E}_{\varepsilon_{n}, h_{n}}\left(t_{n}, \tilde{q}_{n}\right)+\mathcal{D}_{\varepsilon_{n}, h_{n}}\left(q_{n}, \tilde{q}_{n}\right)-\mathcal{E}_{\varepsilon_{n}, h_{n}}\left(t_{n}, q_{n}\right) \geq 0$. Using the limsup estimate in (2.19) gives

$$
0 \leq \limsup _{n \rightarrow \infty} \mathcal{E}_{\varepsilon_{n}, h_{n}}\left(t_{n}, \tilde{q}_{n}\right)+\mathcal{D}_{\varepsilon_{n}, h_{n}}\left(q_{n}, \tilde{q}_{n}\right)-\mathcal{E}_{\varepsilon_{n}, h_{n}}\left(t_{n}, q_{n}\right) \leq \mathcal{E}(t, \tilde{q})+\mathcal{D}(q, \tilde{q})-\mathcal{E}(t, q)
$$

Since $\tilde{q}$ was arbitrary, definition $(2.5)$ gives $q \in \mathcal{S}(t)$.

\subsection{Approximate solutions}

We consider now $\tau>0$, and a partition $0=t_{\tau}^{0}<t_{\tau}^{1}<\ldots<t_{\tau}^{k_{\tau}}=T$ with

$$
t_{i}^{\tau}-t_{i-1}^{\tau} \leq \tau \text { for } i=1, \ldots, k_{\tau} .
$$

We do not assume this partition to be equidistant. Further, we consider an approximation $\left[q_{0}\right]_{h, \varepsilon}$ of the initial condition $q_{0}$ and the following recursive incremental formula: we put $q_{\tau, h, \varepsilon}^{0}=\left[q_{0}\right]_{h, \varepsilon}$ a given initial condition and for $k=1, \ldots, k_{\tau}$ we define $q_{\tau, h, \varepsilon}^{k}$, an approximation of a solution at time $t_{\tau}^{k}$, to be a solution of the minimization problem

$$
\left.\begin{array}{ll}
\text { Minimize } & \mathcal{E}_{\varepsilon, h}\left(t_{\tau}^{k}, q\right)+\mathcal{D}_{\varepsilon, h}\left(z_{\tau, h, \varepsilon}^{k-1}, z\right) \\
\text { subject to } & q=(u, z) \in \mathcal{Q}_{h} .
\end{array}\right\}
$$

We define the approximate solution $q_{\tau, h, \varepsilon}:[0, T] \rightarrow \mathcal{Q}$ as a piecewise constant approximation, namely

$$
q_{\tau, h, \varepsilon}(t):= \begin{cases}q_{\tau, h, \varepsilon}^{k} & \text { for } t_{\tau}^{k-1}<t \leq t_{\tau}^{k}, \quad k=1, \ldots, k_{\tau}, \\ q_{\tau, h, \varepsilon}^{0}=\left[q_{0}\right]_{h, \varepsilon} & \text { for } t=0 .\end{cases}
$$


We also need the "retarded" approximate solution $q_{\tau, h, \varepsilon}^{\mathrm{R}}:[0, T] \rightarrow \mathcal{Q}$ with

$$
q_{\tau, h, \varepsilon}^{\mathrm{R}}(t):= \begin{cases}q_{\tau, h, \varepsilon}^{k} & \text { for } t_{\tau}^{k-1} \leq t<t_{\tau}^{k}, \quad k=1, \ldots, k_{\tau}, \\ q_{\tau, h, \varepsilon}^{k_{\tau}} & \text { for } t=T .\end{cases}
$$

The following result is well known in the theory of rate-independent processes, $c f .[14,41]$. However, as it is relatively easy to prove and contributes considerably to the understanding of the numerical analysis, we provide a full proof.

Proposition 2.3 (discrete stability, energy inequalities, a priori estimates). Let (2.7), the lower semicontinuity (2.8)-(2.10) of the approximate stored and the dissipated energies, and smoothness of external forcing (2.13) hold. Then (2.22) has a solution $q_{\tau, h, \varepsilon}^{k}$ for any $k=1, \ldots, k_{\tau}$ and $q_{\tau, h, \varepsilon}$ is stable in the sense

$$
q_{\tau, h, \varepsilon}(t) \in \mathcal{S}_{\varepsilon, h}\left(t_{\tau}^{k}\right) \quad \text { for any } t \in\left(t_{\tau}^{k-1}, t_{\tau}^{k}\right], \quad k=0, \ldots, k_{\tau}
$$

and satisfies the discrete upper energy inequality

$$
\mathcal{E}_{\varepsilon, h}\left(s, q_{\tau, h, \varepsilon}(s)\right)+\operatorname{Var}_{\mathcal{D}_{\varepsilon, h}}\left(q_{\tau, h, \varepsilon} ; r, s\right)-\mathcal{E}_{\varepsilon, h}\left(r, q_{\tau, h, \varepsilon}(r)\right) \leq \int_{r}^{s} \frac{\partial \mathcal{E}_{\varepsilon, h}}{\partial t}\left(t, q_{\tau, h, \varepsilon}^{\mathrm{R}}(t)\right) \mathrm{d} t
$$

for $r=t_{\tau}^{k_{1}}<s=t_{\tau}^{k_{2}}$ with $k_{1}, k_{2} \in \mathbb{Z} \cap\left[0, k_{\tau}\right]$, as well as a similar discrete lower energy inequality

$$
\mathcal{E}_{\varepsilon, h}\left(s, q_{\tau, h, \varepsilon}(s)\right)+\operatorname{Var}_{\mathcal{D}_{\varepsilon, h}}\left(q_{\tau, h, \varepsilon} ; r, s\right)-\mathcal{E}_{\varepsilon, h}\left(r, q_{\tau, h, \varepsilon}(r)\right) \geq \int_{r}^{s} \frac{\partial \mathcal{E}_{\varepsilon, h}}{\partial t}\left(t, q_{\tau, h, \varepsilon}(t)\right) \mathrm{d} t
$$

for $r=t_{\tau}^{k_{1}}<s=t_{\tau}^{k_{2}}$ but now only for $k_{1}, k_{2} \in \mathbb{N} \cap\left[1, k_{\tau}\right]$. Moreover, we have the a priori estimates

$$
\begin{aligned}
& \mathcal{E}_{\varepsilon, h}\left(t_{\tau}^{k}, q_{\tau, h, \varepsilon}^{k}\right) \leq \mathrm{e}^{c_{1} t_{\tau}^{k}}\left(\mathcal{E}_{\varepsilon, h}\left(0, q_{\tau, h, \varepsilon}^{0}\right)+c_{0}\right)-c_{0} \text { for all } k=0,1, \ldots, k_{\tau}, \\
& \operatorname{Var}_{\mathcal{D}_{\varepsilon, h}}\left(q_{\tau, h, \varepsilon} ; 0, T\right)=\sum_{k=1}^{k_{\tau}} \mathcal{D}_{\varepsilon, h}\left(q_{\tau, h, \varepsilon}^{k-1}, q_{\tau, h, \varepsilon}^{k}\right) \leq \mathrm{e}^{c_{1} T}\left(\mathcal{E}_{\varepsilon, h}\left(0, q_{\tau, h, \varepsilon}^{0}\right)+c_{0}\right) .
\end{aligned}
$$

Proof. The existence of $q_{\tau, h, \varepsilon}^{k}$ solving (2.22) follows from (2.8) and (2.10) via a recursive argument for $k=$ $1, \ldots, k_{\tau}$. Hence $q_{\tau, h, \varepsilon}$ and $q_{\tau, h, \varepsilon}^{\mathrm{R}}$ exist, too.

The discrete stability condition (2.25) follows by using successively that $q_{\tau, h, \varepsilon}^{k}$ is a solution to (2.22) and the triangle inequality $(2.1)$ for $\mathcal{D}_{\varepsilon, h}$ :

$$
\mathcal{E}_{\varepsilon, h}\left(t_{\tau}^{k}, q_{\tau, h, \varepsilon}^{k}\right) \leq \mathcal{E}_{\varepsilon, h}\left(t_{\tau}^{k}, \tilde{q}\right)+\mathcal{D}_{\varepsilon, h}\left(q_{\tau, h, \varepsilon}^{k-1}, \tilde{q}\right)-\mathcal{D}_{\varepsilon, h}\left(q_{\tau, h, \varepsilon}^{k-1}, q_{\tau, h, \varepsilon}^{k}\right) \leq \mathcal{E}_{\varepsilon, h}\left(t_{\tau}^{k}, \tilde{q}\right)+\mathcal{D}_{\varepsilon, h}\left(q_{\tau, h, \varepsilon}^{k}, \tilde{q}\right)
$$

for any $k=1, \ldots, k_{\tau}$.

As to $(2.26)$, we again use that $q_{\tau, h, \varepsilon}^{k}$ solves $(2.22)$ and, comparing it with $q_{\tau, h, \varepsilon}^{k-1}$, we get

$$
\begin{aligned}
\mathcal{E}_{\varepsilon, h}\left(t_{\tau}^{k}, q_{\tau, h, \varepsilon}^{k}\right)-\mathcal{E}_{\varepsilon, h}\left(t_{\tau}^{k-1}, q_{\tau, h, \varepsilon}^{k-1}\right)+\mathcal{D}_{\varepsilon, h}\left(q_{\tau, h, \varepsilon}^{k-1}, q_{\tau, h, \varepsilon}^{k}\right) & \leq \mathcal{E}_{\varepsilon, h}\left(t_{\tau}^{k}, q_{\tau, h, \varepsilon}^{k-1}\right)-\mathcal{E}_{\varepsilon, h}\left(t_{\tau}^{k-1}, q_{\tau, h, \varepsilon}^{k-1}\right) \\
& =\int_{t_{\tau}^{k-1}}^{t_{\tau}^{k}} \frac{\partial \mathcal{E}_{\varepsilon, h}\left(t, q_{\tau, h, \varepsilon}^{k-1}\right)}{\partial t} \mathrm{~d} t
\end{aligned}
$$


Now the estimate (2.26) follows after a summation for $k=k_{1}+1, \ldots, k_{2}$. As to the estimate (2.27), by the stability (2.30) written for $q_{\tau, h, \varepsilon}^{k-1} \tilde{q}=q_{\tau, h, \varepsilon}^{k}$, we find

$$
\begin{aligned}
\mathcal{E}_{\varepsilon, h}\left(t_{\tau}^{k}, q_{\tau, h, \varepsilon}^{k}\right)-\mathcal{E}_{\varepsilon, h}\left(t_{\tau}^{k-1}, q_{\tau, h, \varepsilon}^{k-1}\right)+\mathcal{D}_{\varepsilon, h}\left(q_{\tau, h, \varepsilon}^{k-1}, q_{\tau, h, \varepsilon}^{k}\right) & \geq \mathcal{E}_{\varepsilon, h}\left(t_{\tau}^{k}, q_{\tau, h, \varepsilon}^{k}\right)-\mathcal{E}_{\varepsilon, h}\left(t_{\tau}^{k-1}, q_{\tau, h, \varepsilon}^{k}\right) \\
& =\int_{t_{\tau}^{k-1}}^{t_{\tau}^{k}} \frac{\partial \mathcal{E}_{\varepsilon, h}\left(t, q_{\tau, h, \varepsilon}^{k}\right)}{\partial t} \mathrm{~d} t
\end{aligned}
$$

By a summation for $k=k_{1}+1, \ldots, k_{2}$, we obtain $(2.27)$.

For the a priori estimates we use (2.13) and (2.14) to estimate the right-hand side of (2.31) as

$$
\begin{aligned}
& \int_{t_{\tau}^{k-1}}^{t_{\tau}^{k}} \frac{\partial \mathcal{E}_{\varepsilon, h}\left(t, q_{\tau, h, \varepsilon}^{k-1}\right)}{\partial t} \mathrm{~d} t \leq \int_{t_{\tau}^{k-1}}^{t_{\tau}^{k}} c_{1}\left(\mathcal{E}_{\varepsilon, h}\left(t, q_{\tau, h, \varepsilon}^{k-1}\right)+c_{0}\right) \mathrm{d} t \\
& \leq \int_{t_{\tau}^{k-1}}^{t_{\tau}^{k}} c_{1} \mathrm{e}^{c_{1}\left(t-t_{\tau}^{k-1}\right)}\left(\mathcal{E}_{\varepsilon, h}\left(t_{\tau}^{k-1}, q_{\tau, h, \varepsilon}^{k-1}\right)+c_{0}\right) \mathrm{d} t=\left(\mathrm{e}^{c_{1}\left(t_{\tau}^{k}-t_{\tau}^{k-1}\right)}-1\right)\left(\mathcal{E}_{\varepsilon, h}\left(t_{\tau}^{k-1}, q_{\tau, h, \varepsilon}^{k-1}\right)+c_{0}\right) .
\end{aligned}
$$

We insert this into $(2.31)$ and omit $\mathcal{D}_{\varepsilon, h} \geq 0$ to obtain $\mathcal{E}_{\varepsilon, h}\left(t_{\tau}^{k}, q_{\tau, h, \varepsilon}^{k}\right)+c_{0} \leq \mathrm{e}^{c_{1}\left(t_{\tau}^{k}-t_{\tau}^{k-1}\right)}\left(\mathcal{E}_{\varepsilon, h}\left(t_{\tau}^{k-1}, q_{\tau, h, \varepsilon}^{k-1}\right)+c_{0}\right)$. By induction for $k=1,2, \ldots, k_{\tau}$, assertion (2.28) follows.

Inserting (2.28) into (2.33) and summation for $k=1, \ldots, k_{\tau}$ gives

$$
\begin{aligned}
\int_{0}^{T} \frac{\partial \mathcal{E}_{\varepsilon, h}\left(t, q_{\tau, h, \varepsilon}^{\mathrm{R}}\right)}{\partial t} \mathrm{~d} t & =\sum_{k=1}^{k_{\tau}} \int_{t_{\tau}^{k-1}}^{t_{\tau}^{k}} \frac{\partial \mathcal{E}_{\varepsilon, h}\left(t, q_{\tau, h, \varepsilon}^{k-1}\right)}{\partial t} \mathrm{~d} t \\
& \leq\left(\mathcal{E}_{\varepsilon, h}\left(0, q_{\tau, h, \varepsilon}^{0}\right)+c_{0}\right) \sum_{k=1}^{k_{\tau}}\left(\mathrm{e}^{c_{1} t_{\tau}^{k}}-\mathrm{e}^{c_{1} t_{\tau}^{k-1}}\right)=\left(\mathcal{E}_{\varepsilon, h}\left(0, q_{\tau, h, \varepsilon}^{0}\right)+c_{0}\right)\left(\mathrm{e}^{c_{1} T}-1\right)
\end{aligned}
$$

Inserting this into (2.26) with $r=0=t_{\tau}^{0}$ and $s=T=t_{\tau}^{k_{\tau}}$ and using (2.28) once again yields (2.29).

Remark 2.4 (approximation of initial conditions). Note that (2.32) does not work for $k=1$ because we (intentionally) did not assume "numerical" stability of the approximate initial condition, i.e. $\left[q_{0}\right]_{h, \varepsilon} \in \mathcal{S}_{\varepsilon, h}(0)$ which would only very hardly be guaranteed in concrete numerical schemes. This is also why (2.27) does not hold with $r=0$, unlike (2.26). See also the remark after the statement of Theorem 2.6.

Remark 2.5 (two-sided energy estimate). The two-sided estimate (2.26)-(2.27) is not so crucial for the existence proof itself. In fact, the upper estimate (2.26) is used only for the quite essential a priori bounds (2.28) and (2.29). But the lower bound (2.27) is not really needed, see the remarks right after the statement of Theorem 2.6. However, the two-sided estimate (2.26)-(2.27) has its own relevance as it can be used to check implementation of numerical calculations. Namely, evaluating the terms in (2.26)-(2.27) at each time step and checking a posteriori the estimate (2.26)-(2.27) may detect, e.g., a failure of the minimization procedure, which we have to apply to solve numerically the global optimization problem (2.22) at every current time step; see $[3,4,30,31,47,57,58]$ for numerical results in concrete examples. Violation of $(2.26)$ or $(2.27)$ mean that $q_{\tau, h, \varepsilon}^{k}$ or $q_{\tau, h, \varepsilon}^{k-1}$ cannot be stable, respectively.

\subsection{Convergence of the approximate solutions}

Now we investigate the asymptotics for $(\tau, h, \varepsilon) \rightarrow(0,0,0)$. Like for space discretization, we do not assume the partition of the time interval $[0, T]$ to be nested, but we assume that both time and space discretization refines when $\tau \rightarrow 0$ and $h \rightarrow 0$, respectively. Namely (2.21) for the time discretization, while for the spatial discretization this refinement requirement is implicitly contained in (2.18). Later we will assume explicitly (3.2) to prove (2.19) to obtain (2.18). 
Theorem 2.6. Assume that (2.7)-(2.13), (2.15), (2.17), (2.18), and (2.21) hold and that the initial condition $q_{0}$ is stable:

$$
q_{0} \in \mathcal{S}(0)
$$

and is approximated by $\left[q_{0}\right]_{h, \varepsilon} \in \mathcal{Q}_{h}$ in the sense

$$
\left[q_{0}\right]_{h, \varepsilon} \rightarrow q_{0} \quad \text { and } \quad \mathcal{E}_{\varepsilon}\left(0,\left[q_{0}\right]_{h, \varepsilon}\right) \rightarrow \mathcal{E}\left(0, q_{0}\right)
$$

Then, there exists a subsequence $\left\{\left(\tau_{n}, h_{n}, \varepsilon_{n}\right)\right\}_{n \in \mathbb{N}}$ with $\left(\tau_{n}, h_{n}, \varepsilon_{n}\right) \rightarrow(0,0,0)$ for $n \rightarrow \infty$ satisfying the convergence criterion $h_{n} \leq H\left(\varepsilon_{n}\right)$ from condition $(2.18)$ and a process $q:[0, T] \rightarrow \mathcal{Q}$ being an energetic solution according to Definition 2.1 such that the following holds:

(i) for all $t \in[0, T]$ we have $\mathcal{E}_{\varepsilon_{n}}\left(t, q_{n}(t)\right) \rightarrow \mathcal{E}(t, q(t))$;

(ii) for all $t \in[0, T]$ we have $\operatorname{Var}_{\mathcal{D}_{\varepsilon_{n}}}\left(q_{n} ; 0, t\right) \rightarrow \operatorname{Var}_{\mathcal{D}}(q ; 0, t)$;

(iii) for all $t \in[0, T]$ we have $z_{n}(t) \rightarrow z(t)$ in $\mathcal{Z}$;

(iv) $\frac{\partial}{\partial t} \mathcal{E}_{\varepsilon_{n}}\left(\cdot, q_{n}(\cdot)\right) \rightarrow \frac{\partial}{\partial t} \mathcal{E}(\cdot, q(\cdot))$ in $L^{1}(0, T)$;

(v) for all $t \in[0, T]$ there is a subsequence $\left\{n_{l}\right\}_{l \in \mathbb{N}}$ such that $\lim _{l \rightarrow \infty} u_{n_{l}}(t)=u(t)$ in $\mathcal{U}$; hence $\lim _{l \rightarrow \infty} q_{n_{l}}(t)=q(t)$ in $\mathcal{Q}$,

where we wrote shortly $q_{n}=\left(u_{n}, z_{n}\right)$ for $q_{\tau_{n}, h_{n}, \varepsilon_{n}}^{\mathrm{R}}=\left(u_{\tau_{n}, h_{n}, \varepsilon_{n}}^{\mathrm{R}}, z_{\tau_{n}, h_{n}, \varepsilon_{n}}^{\mathrm{R}}\right)$. If additionally, $\mathcal{E}_{\varepsilon}(t, \cdot, z): \mathcal{U} \rightarrow \mathbb{R}_{\infty}$ is strictly convex, then $(\mathrm{v})$ can be strengthened into $q_{n}(t) \rightarrow q(t)$ in $\mathcal{Q}$ (without further subsequences).

The proof of this result is a direct adaptation of the proof of Theorem 3.4 in [45]. However, we are more general as we do not require $\left[q_{0}\right]_{h, \varepsilon}$ to be stable as in [45], equation (3.6). However, an easy check of the proof shows that the stability of the approximations $\left[q_{0}\right]_{h, \varepsilon}$ is not needed. In fact, the only use of this stability would be that the lower energy estimate (2.27) could be extended to $k_{1}=0$. But this estimate is not used as the lower energy estimate only needed in the limit $(\varepsilon, h)=(0,0)$, see Step 5 in the proof of Theorem 3.4 in [45]. The last statement of the result is proved in [34], see also [46].

Remark 2.7 (weak versus strong convergence). In the Banach-space setting used in the subsequent sections, the typical application is that $\mathcal{Q}=\mathcal{U} \times \mathcal{Z}$ is a Banach space equipped with the weak topology. In such cases the convergences stated in (iii) and (v) mean the weak convergence. However, the additional energy convergence stated in (i) improves this convergence into strong convergence if $\mathcal{E}(t, \cdot)$ is strictly convex, $c f$. [65]. In fact, it is sufficient that the integrand of $\mathcal{E}(t, \cdot)$ is strictly convex in the highest derivatives of $u$ and $z$.

Remark 2.8 (numerical integration). Another approximation of $\mathcal{E}_{\varepsilon}$ and $\mathcal{D}_{\varepsilon}$ involving, e.g., numerical integration can quite easily be incorporated, too. For this, $\mathcal{E}_{\varepsilon}$ and $\mathcal{D}_{\varepsilon}$ in the conditions in Section 2.2 should depend on $h$ in a more complicated way than by adding $\delta_{\mathcal{Q}_{h}}\left(c f\right.$. (2.6)), for instance $\mathcal{E}_{\varepsilon h}$ and $\mathcal{D}_{\varepsilon h}$ may be defined by integration rules rather than by replacing exact integrals. As such a generalization would complicate, in particular Section 3 , and as it will not be used in Section 4, we have omitted it completely.

\section{Linear StruCture}

We consider now the case that $\mathcal{U}$ and $\mathcal{Z}$ are subsets of some reflexive separable Banach spaces $U$ and $Z$, respectively. This enables more detailed considerations.

\subsection{Setting the data and their approximation}

The weak topology, if restricted on bounded convex sets, will play the role of the sequentially compact topology used in Section 2.1 for (2.8)-(2.12), (2.17), and (2.19). Here we will denote it by "w-lim" or " $\stackrel{\mathrm{w}}{\rightarrow}$ " to distinguish it from the norm topology which we will denote by "s-lim" or " $\stackrel{s}{\rightarrow}$ ". In case of non-reflexive spaces having preduals, we could work with weak* topologies instead of the weak ones. For an abstract parameter $h>0$, we consider finite-dimensional subspaces $U_{h} \subset U$ and $Z_{h} \subset Z$. The concrete constructions of $Q_{h}:=U_{h} \times Z_{h}$ 
used in numerical analysis are created by (here an abstract) "(quasi-)interpolation" linear bounded operators $\Pi_{U, h}: U \rightarrow U$ and $\Pi_{Z, h}: Z \rightarrow Z$. We put $\Pi_{h}=\Pi_{U, h} \times \Pi_{Z, h}: Q \rightarrow Q$, and

$$
\mathcal{U}_{h}:=\Pi_{U, h} \mathcal{U}, \quad \mathcal{Z}_{h}:=\Pi_{Z, h} \mathcal{Z}, \quad \mathcal{Q}_{h}:=\mathcal{U}_{h} \times \mathcal{Z}_{h}=\Pi_{h} \mathcal{Q} .
$$

To guarantee the central condition (2.19), we assume the natural basic approximation property that $\Pi_{h}$ converges pointwise to the identity, i.e.

$$
\forall q \in Q: \quad \operatorname{s}_{h \rightarrow 0} \Pi_{h} q=q .
$$

The quasi-interpolation operators need not be conformal with constraints involved implicitly in $\mathcal{U}$ and $\mathcal{Z}$ so that $\mathcal{Q}_{h}$ need not be a subset of $\mathcal{Q}$. As an analytical tool the $\Gamma$-convergence approach allow also for such situations (cf. [45]) but, in order to use the theory from Section 2.1 in a quantitative numerical way, we will always restrict ourselves on "conformal" situations when

$$
\Pi_{U, h} \mathcal{U} \subset \mathcal{U} \quad \text { and } \quad \Pi_{U, h} \mathcal{Z} \subset \mathcal{Z}
$$

i.e. $\mathcal{Q}_{h}=\Pi_{h} \mathcal{Q} \subset \mathcal{Q}$. Possible "nonconformities" can be handled via the penalization parameter $\varepsilon$.

For $X$ another Banach space, it is often useful to consider a mapping $\Xi: U \times Z \rightarrow X$ to describe possible equality constraints of the form $\Xi(u, z)=0$ that may implicitly be involved in the definition of $\mathcal{E}$. Moreover, like in Section 1, we assume the forcing by $f:[0, T] \rightarrow U^{*} \times Z^{*}$ to be given explicitly in $\mathcal{E}$, which covers many applications (except, e.g., "hard-device" loading of mechanical systems through Dirichlet boundary conditions). Then, for $E: U \times Z \rightarrow \mathbb{R}$ we consider

$$
\mathcal{E}(t, u, z):= \begin{cases}E(u, z)-\langle f(t),(u, z)\rangle & \text { if } u \in \mathcal{U}, z \in \mathcal{Z}, \Xi(u, z)=0 \\ +\infty & \text { otherwise. }\end{cases}
$$

The approximate energy deals with possible incompatibility of the finite-dimensional discretization with the equality constraints by a penalization of them $(c f .[53])$ :

$$
\mathcal{E}_{\varepsilon}(t, u, z):= \begin{cases}E(u, z)-\langle f(t),(u, z)\rangle+\frac{1}{\varepsilon}\|\Xi(u, z)\|_{X}^{\alpha} & \text { if } u \in \mathcal{U}, z \in \mathcal{Z}, \\ +\infty & \text { otherwise. }\end{cases}
$$

To satisfy (2.10), we assume a super-linear growth of $E$ to dominate the linear behavior of $\langle f(t), \cdot\rangle$ :

$$
\lim _{\substack{q \in \mathcal{Q} \\\|q\| \rightarrow \infty}} \frac{E(q)}{\|q\|}=+\infty
$$

Obviously, (3.4) and (3.5) yield simply $\frac{\partial}{\partial t} \mathcal{E}(t, q)=\frac{\partial}{\partial t} \mathcal{E}_{\varepsilon}(t, q)=\left\langle\frac{\partial}{\partial t} f(t), q\right\rangle$ and (2.13a) requires

$$
f \in C^{1}\left([0, T] ; Q^{*}\right) .
$$

The coercivity (3.6) with (3.7) ensure also (2.13b), (2.15) and (2.17).

A quite canonical way to induce the dissipation distances in simpler cases is through a degree-1 homogeneous dissipation potentials. For this, we consider $K \subset Z$ a closed convex cone with the vertex at $0, R: Z \rightarrow \mathbb{R}$ a continuous convex degree-1 homogeneous functional, i.e. $R(a \dot{z})=a R(\dot{z})$ for any $\dot{z} \in Z$ and $a \geq 0$. Then we consider the special case of $\mathcal{D}$ defined by

$$
\mathcal{D}\left(z_{1}, z_{2}\right):= \begin{cases}R\left(z_{2}-z_{1}\right) & \text { if } z_{2}-z_{1} \in K \\ +\infty & \text { otherwise }\end{cases}
$$



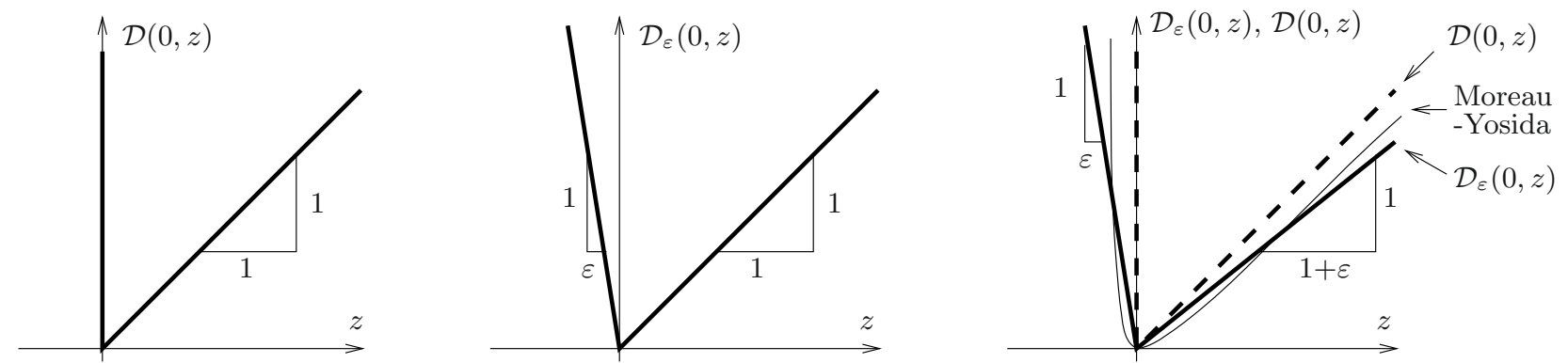

Figure 1. Illustration of $\mathcal{D}(0, \cdot)$ for a special case $Z:=\mathbb{R}, R(z):=|z|, K=[0,+\infty)$ (left) and its approximation by (3.9) (middle) and by (3.10) (right). The last figure depicts also the original $\mathcal{D}(0, \cdot)$ and the Moreau-Yosida approximation for comparison.

Note that $\mathcal{D}\left(z_{1}, z_{1}\right)=0$ and the triangle inequality (2.1) holds. As $R$ is convex and continuous and $K$ convex closed, $\mathcal{D}: Z \times Z \rightarrow \mathbb{R} \cup\{+\infty\}$ is weakly lower semicontinuous.

If $K \neq Z$, then it might be numerically suitable to avoid the unilateral constraints involved by exact penalization by choosing the approximate potential $\mathcal{D}_{\varepsilon}$ in the form

$$
\mathcal{D}_{\varepsilon}\left(z_{1}, z_{2}\right):=R_{\varepsilon}\left(z_{2}-z_{1}\right) \quad \text { where } \quad R_{\varepsilon}(z):=R(z)+\inf _{\hat{z} \in K} \frac{\|z-\hat{z}\|}{\varepsilon} .
$$

Alternatively, we could also consider

$$
R_{\varepsilon}(z):=\inf _{\hat{z} \in K}\left(R(\hat{z})+\frac{\|z-\hat{z}\|^{a}}{a \varepsilon}\right) \quad \text { with } \quad a=1 .
$$

For $a=2$ in (3.10), we would obtain the standard Moreau-Yosida approximation of $R+\delta_{K}$ which is smooth (cf. Fig. 1 (right)) and thus often favorable, but since it fails to be homogeneous degree-1; thus the triangle inequality (2.7) does not hold and its usage would need nontrivial modifications of our theory in Section 2.1. As $K$ is a cone and $a=1$ in (3.10), both $R_{\varepsilon}$ from (3.9) and from (3.10) are again homogeneous degree- 1 functionals for all $\varepsilon>0$, and thus (2.7) holds. As $R$ is convex and continuous and $K$ is convex, $R_{\varepsilon}$ is convex and continuous, implying the weak lower-semicontinuity (2.8) for $R_{\varepsilon}$. Moreover, we always have $R_{\varepsilon} \leq R+\delta_{K}$.

The stability (2.35) of the initial condition $q_{0}$ is, in general, difficult to verify and explicit constructions can be done in very special cases only. Anyhow, there is one universal way how to design a "gentle start", namely taking $q_{0}=\left(u_{0}, z_{0}\right)$ minimizing $\mathcal{E}(0, \cdot)$, i.e. here a solution to the problem

$$
\left.\begin{array}{ll}
\text { minimize } & E(u, z)-\langle f(0),(u, z)\rangle, \\
\text { subject to } & \Xi(u, z)=0, \quad u \in \mathcal{U}, \quad z \in \mathcal{Z} .
\end{array}\right\}
$$

Such a "gentle start" is, in fact, practically the only option applied in engineering simulations.

The other assumptions from Section 2.1 deserve a more detailed proof.

Proposition 3.1 (verification of (2.9), (2.11), and (2.12)). Let $E$ be weakly lower semicontinuous, $\Xi: Q \rightarrow X$ be weakly continuous, and let $K$ be convex and closed, $R$ be convex and also positive on $K \backslash\{0\}$, i.e.

$$
\forall z \in K: \quad z \neq 0 \quad \Rightarrow \quad R(z)>0 \text {. }
$$

Then, (2.9), (2.11), and (2.12) with “ $\rightarrow$ " referring to the weak topology hold.

Proof. In view of (3.4), the condition $\mathcal{E}_{\varepsilon}\left(\theta, q_{h, \varepsilon}\right) \leq a<+\infty$ in $(2.11)$ implies $E\left(q_{h, \varepsilon}\right) \leq C+\left\langle f(\theta), q_{h, \varepsilon}\right\rangle$, and by (3.6) a sequence of $\left\{q_{h, \varepsilon}\right\}_{h, \varepsilon>0}$ must be bounded hence it has a subsequence which converges weakly (recall that we assume reflexivity of $Q)$, which proves (2.11). 
As to (2.9), for $z_{2}-z_{1} \in K$ we have

$$
\begin{aligned}
\liminf _{(h, \varepsilon) \rightarrow(0,0)} \mathcal{D}_{\varepsilon}\left(z_{h, \varepsilon}, \tilde{z}_{h, \varepsilon}\right) & =\liminf _{(h, \varepsilon) \rightarrow(0,0)} R\left(\tilde{z}_{h, \varepsilon}-z_{h, \varepsilon}\right)+\inf _{\tilde{z} \in K} \frac{\left\|\tilde{z}_{h, \varepsilon}-z_{h, \varepsilon}-\hat{z}\right\|}{\varepsilon} \\
& \geq \liminf _{(h, \varepsilon) \rightarrow(0,0)} R\left(\tilde{z}_{h, \varepsilon}-z_{h, \varepsilon}\right) \geq R(\tilde{z}-z)=\mathcal{D}(z, \tilde{z})
\end{aligned}
$$

because $R$ is weakly lower semicontinuous. If $z_{2}-z_{1} \notin K$, then $\inf _{\hat{z} \in K}\|\tilde{z}-z-\hat{z}\|>0$ because $K$ is closed. Using also (3.12), we then have

$$
\liminf _{(h, \varepsilon) \rightarrow(0,0)} \mathcal{D}_{\varepsilon}\left(z_{h, \varepsilon}, \tilde{z}_{h, \varepsilon}\right) \geq \lim _{(h, \varepsilon) \rightarrow(0,0)} \inf _{\tilde{z} \in K} \frac{\left\|z_{h, \varepsilon}-\tilde{z}_{h, \varepsilon}-\hat{z}\right\|}{\varepsilon}=+\infty=\mathcal{D}(z, \tilde{z}) .
$$

As to (2.12), let us distinguish whether $\Xi(q)=0$ or $\Xi(q) \neq 0$. The former case ensures the last equality in the following estimate:

$$
\begin{aligned}
\liminf _{(h, \varepsilon, \theta) \rightarrow(0,0, t)} \mathcal{E}_{\varepsilon}\left(\theta, q_{h, \varepsilon}\right) & =\liminf _{(h, \varepsilon) \rightarrow(0,0)} E\left(q_{h, \varepsilon}\right)-\left\langle f(\theta), q_{h, \varepsilon}\right\rangle+\frac{1}{\varepsilon}\left\|\Xi\left(q_{h, \varepsilon}\right)\right\|_{X}^{\alpha} \\
& \geq \liminf _{(h, \varepsilon, \theta) \rightarrow(0,0, t)} E\left(q_{h, \varepsilon}\right)-\left\langle f(\theta), q_{h, \varepsilon}\right\rangle \geq E(q)-\langle f(t), q\rangle=\mathcal{E}(t, q),
\end{aligned}
$$

where the last inequality is by the weak lower semicontinuity of $E$. This proves that (2.12) holds with respect to the weak topology if $\Xi(q)=0$. In the case $\Xi(q) \neq 0, q_{h, \varepsilon} \stackrel{\mathrm{w}}{\rightarrow} q$ and the weak continuity of $\Xi$ ensures $\liminf \left\|\Xi\left(q_{h, \varepsilon}\right)\right\|_{X} \geq\left\|\mathrm{w}-\lim \Xi\left(q_{h, \varepsilon}\right)\right\|_{X}=\|\Xi(q)\|_{X}>0$. Then, because of the coercivity (3.6) of $E$, we have

$$
\liminf _{\substack{\theta \rightarrow t \\(h, \varepsilon) \rightarrow(0,0)}} \mathcal{E}_{\varepsilon}\left(\theta, q_{h, \varepsilon}\right) \geq \inf _{\substack{\tilde{q} \in Q \\ \theta \in[0, T]}}[E-f(\theta)](\tilde{q})+\lim _{(h, \varepsilon) \rightarrow(0,0)} \frac{1}{\varepsilon}\left\|\Xi\left(q_{h, \varepsilon}\right)\right\|_{X}^{\alpha}=+\infty=\mathcal{E}(t, q) .
$$

In view of the above considerations, we have guaranteed the assumptions needed in Theorem 2.6 except (2.19) and (2.36). This conditions are still to be verified in particular cases, some of them scrutinized in Sections 3.2-3.4.

Remark 3.2 (BV-estimates). Assuming coercivity of $R+\delta_{K}$ on some Banach space $Z_{1} \supset Z$, i.e.

$$
\lim _{z \in K,\|z\|_{Z_{1}} \rightarrow \infty} R(z)=+\infty
$$

together with the degree-1 homogeneity will make (3.12) more specific, namely $\left[R+\delta_{K}\right](z) \geq c\|z\|_{Z_{1}}$ with some $c>0$, hence by (3.8) also $\mathcal{D}\left(q_{1}, q_{2}\right) \geq c\left\|z_{1}-z_{2}\right\|_{Z_{1}}$, and by the definition of "Var" in (2.4b) then also

$$
\operatorname{Var}_{\mathcal{D}}(q ; 0, T) \geq c \operatorname{Var}_{\|\cdot\|_{Z_{1}}}(z ; 0, T) .
$$

In view of Definition (2.4b) applied now with the norm $\|\cdot\|_{Z_{1}}$, the last expression is just the standard total variation and the estimate (2.29) yields boundedness of $z_{\tau, h, \varepsilon}$ and thus also the limit $z$ in the bounded-variation space $\mathrm{BV}\left(0, T ; Z_{1}\right)$.

\subsection{The case $K=Z$}

Let us consider an additional norm $|\cdot|$, which may induce a weaker topology than the canonical norm making $Q$ a Banach space. 
Proposition 3.3 (verification of (2.19) and (2.36) for $K=Z$ ). Let (3.6) and (3.7) hold, and let $\alpha \geq 1$, let $E: Q \rightarrow \mathbb{R}$ in (3.4) be weakly lower semicontinuous and norm continuous, both $\Xi: Q \rightarrow X$ and $R: Z \rightarrow \mathbb{R}$ be weakly continuous, and $K=Z$ (hence $R_{\varepsilon} \equiv R$ ), and $\Xi$ be also Lipschitz continuous with respect to $|\cdot|$, i.e.

$$
\exists \ell_{\Xi} \in \mathbb{R} \quad \forall q_{1}, q_{2} \in \mathcal{Q}: \quad\left\|\Xi\left(q_{1}\right)-\Xi\left(q_{2}\right)\right\|_{X} \leq \ell_{\Xi}\left|q_{1}-q_{2}\right|
$$

and let the operator $\Pi_{h}$ satisfies the convergence-rate estimate

$$
\exists \gamma>0, C \in \mathbb{R} \quad \forall q \in \mathcal{Q}: \quad\left|q-\Pi_{h} q\right| \leq C h^{\gamma}\|q\| .
$$

Then (2.19) and (2.36) with $q_{0} \in \mathcal{S}(0)$ are satisfied, the last two conditions relying on the convergence criterion

$$
H(\varepsilon)=o\left(\varepsilon^{\frac{1}{\alpha \gamma}}\right) \quad \text { and with } \quad \tilde{q}_{h, \varepsilon} \stackrel{\mathrm{s}}{\rightarrow} \tilde{q} .
$$

Proof. Let us prove (2.19). For any $\tilde{q} \in Q$, with $\Xi(\tilde{q})=0$, by (3.18) and (3.19), we have

$$
\left\|\Xi\left(\Pi_{h} \tilde{q}\right)\right\|_{X}=\left\|\Xi\left(\Pi_{h} \tilde{q}\right)-\Xi(\tilde{q})\right\|_{X} \leq \ell \Xi\left|\tilde{q}-\Pi_{h} \tilde{q}\right| \leq C \ell_{\Xi} h^{\gamma}\|\tilde{q}\| .
$$

For $(h, \varepsilon) \rightarrow(0,0)$ with $h \leq H(\varepsilon)$ with $H$ from (3.20) we therefore have

$$
\frac{1}{\varepsilon}\left\|\Xi\left(\Pi_{h} \tilde{q}\right)\right\|_{X}^{\alpha} \leq C^{\alpha} \ell_{\Xi}^{\alpha} \frac{h^{\alpha \gamma}}{\varepsilon}\|\tilde{q}\|^{\alpha} \rightarrow 0 .
$$

We put $\tilde{q}_{h, \varepsilon}:=\Pi_{h} \tilde{q}$ for (2.19); note that, in fact, we do not need any explicit dependence on $\varepsilon$ except that we assume $h \leq H(\varepsilon)$. As $E$ is strongly continuous and, by $(3.2), \tilde{q}_{h, \varepsilon} \stackrel{\mathrm{s}}{\rightarrow} \tilde{q}$, and as $R$ is weakly continuous and $q_{h, \varepsilon} \stackrel{\mathrm{w}}{\rightarrow} q$ is assumed in (2.19), it holds

$$
\begin{aligned}
\lim _{\substack{h \leq H(\varepsilon) \\
(\varepsilon, h) \rightarrow(0,0)}} \mathcal{E}_{\varepsilon}\left(\theta, \tilde{q}_{h, \varepsilon}\right)+\mathcal{D}\left(q_{h, \varepsilon}, \tilde{q}_{h, \varepsilon}\right) & =\lim _{\substack{h \leq H(\varepsilon) \\
(\varepsilon, h) \rightarrow(0,0)}} E\left(\tilde{q}_{h, \varepsilon}\right)-\left\langle f(\theta), \tilde{q}_{h, \varepsilon}\right\rangle+R\left(\tilde{q}_{h, \varepsilon}-q_{h, \varepsilon}\right)+\frac{1}{\varepsilon}\left\|\Xi\left(\Pi_{h} \tilde{q}\right)\right\|_{X}^{\alpha} \\
& =E(\tilde{q})-\langle f(t), \tilde{q}\rangle+R(\tilde{q}-q)=\mathcal{E}(t, \tilde{q})+\mathcal{D}(q, \tilde{q})
\end{aligned}
$$

whenever $\Xi(\tilde{q})=0$. Combining this with (2.12), we obtain (2.19) for $\Xi(\tilde{q})=0$. If $\Xi(\tilde{q}) \neq 0$, then due to the definition (3.4) the right-hand side in (2.19) is $+\infty$ and (2.19) is fulfilled trivially.

The stability of $q_{0}$ considered in Theorem 2.6 implies $\mathcal{E}\left(0, q_{0}\right)<+\infty$, and then the assumption (2.36) is fulfilled if one chooses $\left[q_{0}\right]_{h, \varepsilon}:=\Pi_{h} q_{0}$ in (2.36). Indeed, $\left[q_{0}\right]_{h, \varepsilon} \stackrel{\mathrm{s}}{\rightarrow} q_{0}$ for $h \rightarrow 0$ just by (3.2) and then also $\mathcal{E}_{\varepsilon}\left(0,\left[q_{0}\right]_{h, \varepsilon}\right)=E\left(\Pi_{h} q_{0}\right)+\frac{1}{\varepsilon}\left\|\Xi\left(\Pi_{h} q_{0}\right)\right\|_{X}^{\alpha}-\left\langle f(0), \Pi_{h} q_{0}\right\rangle \rightarrow E\left(q_{0}\right)-\left\langle f(0), q_{0}\right\rangle=\mathcal{E}\left(0, q_{0}\right)$ because $E$ is assumed norm continuous and because, since the finite energy of $q_{0}$ implies $\Xi\left(q_{0}\right)=0$, we can employ the estimate (3.22) for $h \leq H(\varepsilon)$.

\subsection{The case $K \varsubsetneqq Z$}

Certain applications to unidirectional processes (like damage, delamination, debonding, or hardening in plasticity or in ferromagnets) require modeling with $K \varsubsetneqq Z$. This needs further finer investigations for which we consider some topology $\sigma$ on $U \times Z$ which is finer than the weak one and coarser than the norm one; see the particular examples in Section 4.

Proposition 3.4 (unconditional convergence for $K \varsubsetneqq Z$ ). Let $E: Q \rightarrow \mathbb{R}$ be weakly lower semicontinuous and $\sigma$-continuous. Assume both $R: Z \rightarrow \mathbb{R}$ and $\Xi: Q \rightarrow X$ be weakly continuous, and let (3.7), and that the following attainability condition, expressing certain consistency of the discretization with the constraints given 
by $\Xi$ and $K$, hold:

$$
\begin{aligned}
& \forall q, \tilde{q} \in \mathcal{Q}, \quad \Xi(q)=0, \quad \tilde{q}-q \in K, \quad \Xi(\tilde{q})=0, \quad \forall q_{h} \in \mathcal{Q}_{h}, \quad q_{h} \stackrel{\mathrm{w}}{\rightarrow} q \\
& \exists \tilde{q}_{h} \in \mathcal{Q}_{h}: \quad \tilde{q}_{h} \stackrel{\sigma}{\rightarrow} \tilde{q}, \quad\left\|\Xi\left(\tilde{q}_{h}\right)\right\|_{X} \leq\left\|\Xi\left(q_{h}\right)\right\|_{X}, \quad \tilde{q}_{h}-q_{h} \in K .
\end{aligned}
$$

Then (2.19) with $\mathcal{D}_{\varepsilon, h}$ from (3.9) is satisfied, now with $H \equiv 1$, i.e. "unconditionally". Moreover, the qualification (2.36) of the stable initial condition $q_{0}$ holds if

$$
\exists q_{0 h} \in \mathcal{Q}_{h}: \quad \Xi\left(q_{0 h}\right)=0 \quad \& \quad q_{0 h} \stackrel{\sigma}{\rightarrow} q_{0} .
$$

Proof. The a priori bound $\mathcal{E}_{\varepsilon, h}\left(\theta, q_{h, \varepsilon}\right) \leq C$ assumed in (2.19) means

$$
\frac{1}{\varepsilon}\left\|\Xi\left(q_{h, \varepsilon}\right)\right\|_{X}^{\alpha} \leq C-E\left(q_{h, \varepsilon}\right)+\left\langle f(\theta), q_{h, \varepsilon}\right\rangle \leq C+\sup _{q \in Q, \theta \in[0, T]}[f(\theta)-E](q)<+\infty
$$

due to (3.6) so that $\left\|\Xi\left(q_{h, \varepsilon}\right)\right\|_{X}=\mathcal{O}\left(\varepsilon^{1 / \alpha}\right)$. In the limit therefore $\Xi(q)=0$ because $\Xi$ is assumed weakly continuous. Thus we take $q_{h, \varepsilon}$ from (2.19) for $q_{h}$ in (3.24). As (2.19) is trivially satisfied if $\Xi(\tilde{q}) \neq 0$ because the right-hand side in $(2.19)$ is $+\infty$, we can consider only $\Xi(\tilde{q})=0$. Then we can take $\tilde{q}_{h}$ from $(3.24)$ for $\tilde{q}_{h, \varepsilon}$ in (2.19). Note that $\tilde{q}_{h, \varepsilon}-q_{h, \varepsilon} \in K$ in (3.24) ensures $\mathcal{D}_{\varepsilon, h}\left(q_{h, \varepsilon}, \tilde{q}_{h, \varepsilon}\right)=R\left(q_{h, \varepsilon}-\tilde{q}_{h, \varepsilon}\right)$ due to Definition (3.9) and by the assumed weak continuity of $R$ and closeness and convexity of $K$, we have

$$
\lim _{(h, \varepsilon) \rightarrow(0,0)} \mathcal{D}_{\varepsilon, h}\left(q_{h, \varepsilon}, \tilde{q}_{h, \varepsilon}\right)=\lim _{(h, \varepsilon) \rightarrow(0,0)} R\left(\tilde{q}_{h, \varepsilon}-q_{h, \varepsilon}\right)=R(\tilde{q}-q)=\mathcal{D}(q, \tilde{q})
$$

Then, using the $\sigma$-continuity and weak lower semicontinuity of $E$ the continuity of $f$ (see $(3.7)$ ), and $\left\|\Xi\left(\tilde{q}_{h, \varepsilon}\right)\right\|_{X} \leq$ $\left\|\Xi\left(q_{h, \varepsilon}\right)\right\|_{X}($ see $(3.24))$, we obtain

$$
\begin{aligned}
& \quad \limsup _{(h, \varepsilon, \theta) \rightarrow(0,0, t)}\left(\mathcal{E}_{\varepsilon, h}\left(\theta, \tilde{q}_{h, \varepsilon}\right)+\mathcal{D}_{\varepsilon, h}\left(q_{h, \varepsilon}, \tilde{q}_{h, \varepsilon}\right)-\mathcal{E}_{\varepsilon, h}\left(\theta, q_{h, \varepsilon}\right)\right)= \\
& \quad \limsup _{(h, \varepsilon, \theta) \rightarrow(0,0, t)}\left(E\left(\tilde{q}_{h, \varepsilon}\right)-\left\langle f(\theta), \tilde{q}_{h, \varepsilon}-q_{h, \varepsilon}\right\rangle+\frac{1}{\varepsilon}\left\|\Xi\left(\tilde{q}_{h, \varepsilon}\right)\right\|_{X}^{\alpha}+\mathcal{D}\left(q_{h, \varepsilon}, \tilde{q}_{h, \varepsilon}\right)-E\left(q_{h, \varepsilon}\right)-\frac{1}{\varepsilon}\left\|\Xi\left(\tilde{q}_{h, \varepsilon}\right)\right\|_{X}^{\alpha}\right) \\
& \leq \limsup _{(h, \varepsilon, \theta) \rightarrow(0,0, t)}\left(E\left(\tilde{q}_{h, \varepsilon}\right)-\left\langle f(\theta), \tilde{q}_{h, \varepsilon}-q_{h, \varepsilon}\right\rangle+\mathcal{D}\left(q_{h, \varepsilon}, \tilde{q}_{h, \varepsilon}\right)-E\left(q_{h, \varepsilon}\right)\right) \\
& \quad=\lim _{(h, \varepsilon, \theta) \rightarrow(0,0, t)}\left(E\left(\tilde{q}_{h, \varepsilon}\right)-\left\langle f(\theta), \tilde{q}_{h, \varepsilon}-q_{h, \varepsilon}\right\rangle+\mathcal{D}\left(q_{h, \varepsilon}, \tilde{q}_{h, \varepsilon}\right)\right)-\liminf _{(h, \varepsilon) \rightarrow(0,0)} E\left(q_{h, \varepsilon}\right) \\
& \leq E(\tilde{q})-\langle f(t), \tilde{q}-q\rangle+\mathcal{D}(q, \tilde{q})-E(q)=\mathcal{E}(t, \tilde{q})+\mathcal{D}(q, \tilde{q})-\mathcal{E}(t, q)
\end{aligned}
$$

Eventually, we are to prove (2.36) provided (3.25) and provided $q_{0} \in \mathcal{S}(0)$; the last inclusion implies $\mathcal{E}\left(0, q_{0}\right)<+\infty$ which here further implies $\Xi\left(q_{0}\right)=0$. Then, with $\left[q_{0}\right]_{h, \varepsilon}:=q_{0 h}$ in $(3.25)$, it holds

$$
\mathcal{E}_{\varepsilon, h}\left(0,\left[q_{0}\right]_{h, \varepsilon}\right)=E\left(q_{0 h}\right)-\left\langle f(0), q_{0 h}\right\rangle \rightarrow E\left(q_{0}\right)-\left\langle f(0), q_{0}\right\rangle=\mathcal{E}\left(0, q_{0}\right)
$$

as required in (2.36) because $\mathcal{E}$ is assumed $\sigma$-continuous. Note that the last equality in (3.29) relies on $\Xi\left(q_{0}\right)=0$ for which $\sigma$-continuity of $\Xi$ is needed; in fact, we assumed even weak continuity of $\Xi$.

\subsection{The case $K \varsubsetneqq Z$ and "semiquadratic" $E$}

Some applications exhibits the "main" part of the stored energy E quadratic in terms of the dissipating variable $z$ in the sense

$$
\begin{array}{ll}
E(u, z):=\frac{1}{2}\langle B z, z\rangle+E_{0}(u, z), \quad B: Z \rightarrow Z^{*} \text { linear and bounded, } \\
\\
E_{0}: U \times Z \rightarrow \mathbb{R}(\mathrm{s} \times \mathrm{w}) \text {-continuous. }
\end{array}
$$


In smooth cases, this corresponds to problems governed by "semilinear" mappings $E^{\prime}(q)=\left(\begin{array}{cc}0 & 0 \\ 0 & B\end{array}\right)+E_{0}^{\prime}(q)$. Such problems are well fitted for unconditional convergence under some particular circumstances.

As to (2.36), we can guarantee it again through (3.25) now with $\sigma$ the strong topology to have the quadratic term in (3.30) continuous. The verification of (2.19) is now more sophisticated:

Proposition 3.5 ("semiquadratic" case: unconditional convergence). Let (3.7) and (3.30) hold, $R$ be continuous, let further $\Xi$ be independent of $u$, affine and continuous, i.e. in the form $\Xi(u, z)=\Xi_{0}(z)+\xi$ with $\xi \in X$, and $\Xi_{0} \in \mathcal{L}(Z, X)$ compatible with the discretization operator $\Pi_{Z, h}$ in the sense that $\Pi_{Z, h}\left(\operatorname{Ker} \Xi_{0}\right) \subset \operatorname{Ker} \Xi_{0}$. Let also $\mathcal{Z}+K \subset \mathcal{Z}$, and the cone $K$ be compatible with $\Pi_{Z, h}$ in the sense that $\Pi_{Z, h} K \subset K$. Then (2.19) with $H \equiv 1$ holds.

Proof. We will prove (2.19) by using Proposition 3.4 and for this we will verify (3.24) with $\sigma$ being the strong $\times$ weak topology on $U \times Z$. The recovery element $\tilde{q}_{h}$ in (3.24) can be chosen simply as

$$
\begin{aligned}
& \tilde{u}_{h}:=\Pi_{U, h} \tilde{u}, \\
& \tilde{z}_{h}:=z_{h}+\Pi_{Z, h}(\tilde{z}-z) .
\end{aligned}
$$

It holds $\tilde{q}_{h} \in \mathcal{Q}_{h}$; indeed, $\tilde{u}_{h} \in \mathcal{U}_{h}$ just by Definitions (3.1) and (3.31a) while $\tilde{z}_{h} \in \mathcal{Z}_{h}$ because $\tilde{z}-z \in K$, assumed in (3.24), implies $\tilde{z}_{h}-z_{h}=\Pi_{Z, h}(\tilde{z}-z) \in \Pi_{Z, h} K$ and further $\mathcal{Z}+K \subset \mathcal{Z}$ implies $\mathcal{Z}_{h}=\Pi_{Z, h} \mathcal{Z} \supset$ $\Pi_{Z, h}(\mathcal{Z}+K)=\mathcal{Z}_{h}+\Pi_{Z, h} K$ and eventually $z_{h} \in \mathcal{Z}_{h}$ is assumed in (3.24), hence $\tilde{z}_{h} \in \mathcal{Z}_{h}$ indeed follows.

Also, the inequality $\left\|\Xi\left(q_{h}\right)\right\|_{X} \leq\left\|\Xi\left(\tilde{q}_{h}\right)\right\|_{X}$ in (3.24) follows from

$$
\Xi\left(\tilde{q}_{h}\right)=\Xi_{0} \tilde{z}_{h}+\xi=\Xi_{0}\left(z_{h}+\Pi_{Z, h}(\tilde{z}-z)\right)+\xi=\Xi\left(q_{h}\right)
$$

because $\Xi_{0}\left(\Pi_{Z, h}(z-\tilde{z})\right)=0$ holds. Indeed, $\Xi(\tilde{q})=0$ is also explicitly assumed in (3.24) while $\Xi(q)=0$ follows from $q_{h} \stackrel{\mathrm{W}}{\rightarrow} q$ assumed in (3.24) by the continuity of $\Xi$, and therefore $\Xi_{0}(z-\tilde{z})=\Xi(q)-\Xi(\tilde{q})=0$, hence $z-\tilde{z} \in \operatorname{Ker} \Pi_{0}$, and by the assumed compatibility $\Pi_{Z, h}\left(\operatorname{Ker} \Xi_{0}\right) \subset \operatorname{Ker} \Xi_{0}$ also $\Pi_{Z, h}(z-\tilde{z}) \in \operatorname{Ker} \Xi_{0}$, hence eventually $\Xi_{0}\left(\Pi_{Z, h}(z-\tilde{z})\right)=0$. Then also, by using also (3.2), it holds

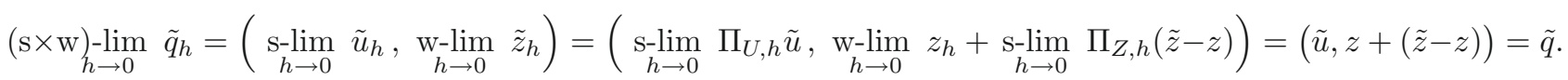

Although for $\sigma=\mathrm{s} \times \mathrm{w}$ the energy $E$ itself need not be $\sigma$-continuous like in Proposition 3.4, in the case (3.30) it is however possible to pass to the limit in the difference $\mathcal{E}\left(\theta, \tilde{q}_{h}\right)-\mathcal{E}\left(\theta, q_{h}\right)$ by using (3.32) and the binomial formula:

$$
\begin{aligned}
\mathcal{E}_{\varepsilon}\left(\theta, \tilde{q}_{h}\right)-\mathcal{E}_{\varepsilon}\left(\theta, q_{h}\right) & =\mathcal{E}\left(\theta, \tilde{q}_{h}\right)+\frac{1}{\varepsilon}\left\|\Xi\left(\tilde{q}_{h}\right)\right\|_{X}^{\alpha}-\mathcal{E}\left(\theta, q_{h}\right)-\frac{1}{\varepsilon}\left\|\Xi\left(q_{h}\right)\right\|_{X}^{\alpha} \\
& =\mathcal{E}\left(\theta, \tilde{q}_{h}\right)-\mathcal{E}\left(\theta, q_{h}\right) \\
& =\frac{1}{2}\left\langle B \tilde{z}_{h}, \tilde{z}_{h}\right\rangle-\frac{1}{2}\left\langle B z_{h}, z_{h}\right\rangle+E_{0}\left(\tilde{q}_{h}\right)-E_{0}\left(q_{h}\right)-\left\langle f(\theta), \tilde{q}_{h}-q_{h}\right\rangle \\
& =\frac{1}{2}\left\langle B\left(\tilde{z}_{h}-z_{h}\right), \tilde{z}_{h}+z_{h}\right\rangle+E_{0}\left(\tilde{q}_{h}\right)-E_{0}\left(q_{h}\right)-\left\langle f(\theta), \tilde{q}_{h}-q_{h}\right\rangle \\
& \rightarrow \frac{1}{2}\langle B(\tilde{z}-z), \tilde{z}+z\rangle+E_{0}(\tilde{q})-E_{0}(q)-\langle f(t), \tilde{q}-q\rangle \\
& =\frac{1}{2}\langle B \tilde{z}, \tilde{z}\rangle-\frac{1}{2}\langle B z, z\rangle+E_{0}(\tilde{q})-E_{0}(q)-\langle f(t), \tilde{q}-q\rangle=\mathcal{E}(t, \tilde{q})-\mathcal{E}(t, q) .
\end{aligned}
$$

For the limit passage it was important that $\tilde{z}_{h}-z_{h}=\Pi_{Z, h}(\tilde{z}-z) \stackrel{\mathrm{s}}{\rightarrow} \tilde{z}-z$ because of (3.2) so that

$$
\left\langle B\left(\tilde{z}_{h}-z_{h}\right), \tilde{z}_{h}+z_{h}\right\rangle \rightarrow\langle B(\tilde{z}-z), \tilde{z}+z\rangle
$$


TABLE 1. Organization and features of the examples presented in Section 4.

\begin{tabular}{|c||c|c|c|c|}
\hline Process / Section & $\begin{array}{c}\text { Unidirectional } \\
(\text { i.e. } K \varsubsetneqq Z)\end{array}$ & $\begin{array}{c}\text { Constraints } \\
(\text { i.e. } X \neq\{0\})\end{array}$ & $\begin{array}{c}\text { Quadratic } \\
\text { energy } E\end{array}$ & $\begin{array}{c}\text { Proposition } \\
\text { used }\end{array}$ \\
\hline $\begin{array}{c}\text { Plasticity with hardening } \\
\text { at small strains / } 4.2\end{array}$ & + & - & + & 3.5 \\
\hline $\begin{array}{c}\text { Phase transformation: } \\
\text { mixture approach / } 4.3\end{array}$ & - & - & - & $3.4(\sigma=\mathrm{s})$ \\
\hline $\begin{array}{c}\text { Phase transformation: } \\
\text { non-mixture approach/4.4 }\end{array}$ & - & + & - & $3.4(\sigma=\mathrm{s})$ \\
\hline Damage / 4.5 & + & - & \pm & 3.5 \\
\hline Debonding / 4.6 & + & - & - & $3.4\left(\sigma=\mathrm{s} \times \mathrm{w}^{*}\right)$ \\
\hline Magnetostriction / 4.7 & - & + & - & 3.3 \\
\hline
\end{tabular}

because $\tilde{z}_{h}+z_{h} \stackrel{\mathrm{w}}{\rightarrow} z+\tilde{z}$. We have $\tilde{z}_{h}-z_{h}=\Pi_{Z, h}(\tilde{z}-z) \in \Pi_{Z, h} K \subset K$. Then, in view of the definition in (3.8) and the strong continuity of $R$, we have

$$
\begin{aligned}
\lim _{(\varepsilon, h) \rightarrow(0,0)} \mathcal{D}_{\varepsilon}\left(q_{h}, \tilde{q}_{h}\right) & =\lim _{(\varepsilon, h) \rightarrow(0,0)} R_{\varepsilon}\left(\tilde{z}_{h}-z_{h}\right)=\lim _{h \rightarrow 0} R\left(\tilde{z}_{h}-z_{h}\right) \\
& =\lim _{h \rightarrow 0} R\left(\Pi_{Z, h}(\tilde{z}-z)\right)=R\left(\lim _{h \rightarrow 0} \Pi_{Z, h}(\tilde{z}-z)\right)=R(\tilde{z}-z)=\mathcal{D}(q, \tilde{q}) .
\end{aligned}
$$

By (3.33) and (3.35), we can pass to the limit in (3.28). Thus (2.19) with $H \equiv 1$ is proved in this case, too.

Remark 3.6 (no penalization). In case of the unconditional convergence, one can consider a numerical scheme with $\varepsilon=0$, i.e. with the original $\mathcal{E}$ and $\mathcal{D}$ instead of $\mathcal{E}_{\varepsilon, h}$ and $\mathcal{D}_{\varepsilon, h}$. The corresponding incremental problem might then involve unilateral constraint; $c f$. also Remark 4.3.

\section{Particular EXAmples in CONTINUUm MeCHANics}

The doubly-nonlinear inclusion (2.2) is a framework for description of so-called generalized standard materials with internal parameters as introduced by Halphen and Nguyen [21] in those cases where convexity of stored and dissipated energies can be expected and inertial effects can be neglected. Here we have in mind various inelastic rate-independent processes in such materials having possibly a nonconvex stored energy. The following examples illustrate how the general theory applies in particular situations, $c f$. Table 1 for a survey. As a byproduct of the presented numerical theory, we obtain analytical existence/convergence results which have not yet been derived in literature. For the sake of explanatory lucidity, we confine ourselves to rather conventional models from continuum mechanics although some less conventional models (e.g. those involving a microstructure described by so-called Young measures, see [31,55-57]) allow for such numerical analysis, too. In Section 4.7 we present a combination of mechanical and ferromagnetic effects, i.e. magnetostriction with hysteretic effects, but the combination with ferroelectric effects, i.e. piezoelectricity with hysteresis (see [43]), or even purely nonmechanical rate-independent models developed in ferromagnetics (e.g. $[55,56,66,67])$ and ferroelectrics (e.g. [25, $52,60]$ ) could be treated similarly. We neglect any temperature dependence or, in other words, if there is a possible dependence of data on temperature, we consider sufficiently slow processes so that the released heat due to dissipative processes can efficiently be transferred away to allow for considering isothermal processes.

\subsection{Sketch of continuum mechanics of deformable bodies}

We assume a specimen occupying in its reference configuration a bounded domain $\Omega \subset \mathbb{R}^{3}$. As usual, $y: \Omega \rightarrow \mathbb{R}^{3}$ denotes the deformation and $u: \Omega \rightarrow \mathbb{R}^{3}$ the displacement, related to each other by $y(x)=x+u(x)$, 
$x \in \Omega$. Hence the deformation gradient equals $F=\nabla y=\mathbb{I}+\nabla u$ with $\mathbb{I} \in \mathbb{R}^{3 \times 3}$ being the identity matrix and $\nabla$ is the gradient operator. For simplicity, we will treat only the soft-device loading realized through traction (Neumann or Robin-type) boundary conditions. The state of the material and possibly also of boundary conditions is assumed to depend on (a set of) certain parameters $z$ that may evolve in time in a rate-independent manner. Then naturally $U$ and $Z$ used before will be the spaces of $u$ 's and of $z$ 's, respectively.

The specific energy stored in the inter-atomic links in the homogeneous (possibly anisotropic) continuum $\hat{\varphi}=\hat{\varphi}(F, z)$ is phenomenologically described as a function of the deformation gradient $F$ and the mentioned variable $z \in \mathbb{R}^{m}$. Mostly the vector $z \in Z_{0} \subset \mathbb{R}^{m}$ in not directly accessible for a macroscopical loading (for an exception see Sect. 4.7) and will thus play the role of internal parameters. The frame-indifference, i.e. $\hat{\varphi}(F, z)=\hat{\varphi}(R F, z)$ for any $R \in \mathrm{SO}(3)=$ the group of orientation-preserving rotations, requires that $\hat{\varphi}(\cdot, z)$ in fact depends only on the (right) Cauchy-Green stretch tensor

$$
F^{\top} F=(\mathbb{I}+\nabla u)^{\top}(\mathbb{I}+\nabla u)=\mathbb{I}+(\nabla u)^{\top}+\nabla u+(\nabla u)^{\top} \nabla u .
$$

An important property of $\hat{\varphi}(\cdot, z)$ is quasiconvexity, which means $\varphi(A, z) \leq \inf _{u \in W_{0}^{1, p}\left(\Omega ; \mathbb{R}^{3}\right)} \int_{\Omega} \varphi(A+\nabla u, z) \mathrm{d} x$ for any $A \in \mathbb{R}^{3 \times 3}$. The following assertion modifies the celebrated result by Acerbi and Fusco [1]:

Lemma 4.1. Let $\varphi: \mathbb{R}^{3 \times 3} \times \mathbb{R}^{m} \rightarrow \mathbb{R}$ be continuous, $\varphi(\cdot, z)$ quasiconvex, $p, p_{1} \in(1,+\infty)$ and, for some $c_{2} \geq c_{1}>0$,

$$
\forall A \in \mathbb{R}^{3 \times 3} \quad \forall z \in Z_{0}: \quad c_{1}\left(|A|^{p}+|z|^{p_{1}}-1\right) \leq \varphi(A, z) \leq c_{2}\left(1+|A|^{p}+|z|^{p_{1}}\right) .
$$

Then the functional $(u, z) \mapsto \int_{\Omega} \varphi(\nabla u, z) \mathrm{d} x$ is $(w \times s)$-lower semicontinuous on $W^{1, p}\left(\Omega ; \mathbb{R}^{3}\right) \times\left\{z \in L^{p_{1}}\left(\Omega ; \mathbb{R}^{m}\right)\right.$; $z(\cdot) \in Z_{0}$ a.e. on $\left.\Omega\right\}$.

Sketch of the proof. By coercivity, we do not need to distinguish between sequential and topological lower semicontinuity.

Let us take a sequence $\left\{\left(u_{n}, z_{n}\right)\right\}_{n \in \mathbb{N}}(\mathrm{w} \times \mathrm{s})$-converging to $(u, z)$. Then $\left(\nabla u_{n}, z_{n}\right)(\mathrm{w} \times \mathrm{s})$-converges to $(\nabla u, z)$ in $L^{p}\left(\Omega ; \mathbb{R}^{3 \times 3}\right) \times L^{p_{1}}\left(\Omega ; \mathbb{R}^{m}\right)$. Also, selecting a suitable subsequence, it generates (a set) of $L^{p} \times L^{p_{1}}$ Young measures of the form $\nu \otimes \mu_{z}$ where $\mu_{z}=\left\{\delta_{z(x)}\right\}_{x \in \Omega}$ with $\delta_{z(x)}$ denoting here the Dirac distribution on $\mathbb{R}^{m}$ supported at $z(x) ; c f$. [48], Corollary 3.4. This means, in terms of a mentioned subsequence, that

$$
\lim _{n \rightarrow \infty} \int_{\Omega} v\left(\nabla u_{n}, z_{n}\right) \mathrm{d} x=\int_{\Omega} \int_{\mathbb{R}^{3 \times 3} \times \mathbb{R}^{m}} v(A, r)\left[\nu_{x} \otimes \delta_{z(x)}\right](\mathrm{d} A \times \mathrm{d} r) \mathrm{d} x=\int_{\Omega} \int_{\mathbb{R}^{3 \times 3}} v(A, z(x)) \nu_{x}(\mathrm{~d} A) \mathrm{d} x
$$

for any $v$ continuous of a growth less than $p$ in the $A$-variable, while for $\varphi$ continuous satisfying (4.2) we have

$$
\liminf _{n \rightarrow \infty} \int_{\Omega} \varphi\left(\nabla u_{n}, z_{n}\right) \mathrm{d} x \geq \int_{\Omega} \int_{\mathbb{R}^{3 \times 3}} \varphi(A, z(x)) \nu_{x}(\mathrm{~d} A) \mathrm{d} x ;
$$

cf. [49], Theorem 3.2. As $\nu_{x}$ is a gradient $L^{p}$-Young measure with $\int_{\mathbb{R}^{3 \times 3}} A \nu_{x}(\mathrm{~d} A)=\nabla u(x)$ for a.a. $x \in \Omega$, and as $\varphi(\cdot, z(x))$ is quasiconvex, for a.a. $x \in \Omega$ it holds

$$
\int_{\mathbb{R}^{3 \times 3}} \varphi(A, z(x)) \nu_{x}(\mathrm{~d} A) \geq \varphi\left(\int_{\mathbb{R}^{3 \times 3}} A \nu_{x}(\mathrm{~d} A), z(x)\right)=\varphi(\nabla u(x), z(x)) .
$$

see [29,49]. Combining (4.4) and (4.5) yields $\liminf _{n \rightarrow \infty} \int_{\Omega} \varphi\left(\nabla u_{n}, z_{n}\right) \mathrm{d} x \geq \int_{\Omega} \varphi(\nabla u(x), z(x)) \mathrm{d} x$. As the Young measure is not involved in the last estimate at all, this estimate holds, in fact, for the whole original sequence. 
An example of a frame-indifferent quasiconvex (in fact even polyconvex, i.e. convex in terms of $F$ and its determinant and cofactors) energy $\hat{\varphi}(F, z):=\tilde{\varphi}(F)$ satisfying (4.2) is the Ogden-type material

$$
\varphi(F, z)=\alpha_{1} \operatorname{tr}\left(F^{\top} F-\mathbb{I}\right)^{p / 2}+\alpha_{2}\left|\operatorname{tr}\left(\operatorname{cof}\left(F^{\top} F\right)-\mathbb{I}\right)\right|^{p_{0}}+\phi_{0}(\operatorname{det}(F))
$$

here $\alpha_{1}, \alpha_{2}>0, p \geq 3, p_{0} \leq p / 2, \phi_{0}$ is a convex function of at most $p / 3$ growth, and finally $\operatorname{tr}(\cdot)$ in $(4.6)$ denotes the trace of a matrix.

As $F=\mathbb{I}+\nabla u$, we can express the specific stored energy in terms of the displacement gradient as

$$
\varphi=\varphi(\nabla u, z)=\hat{\varphi}(\mathbb{I}+\nabla u, z)
$$

The Piola-Kirchhoff stress $\sigma: \mathbb{R}^{3 \times 3} \rightarrow \mathbb{R}^{3 \times 3}$ is given by $\sigma=\varphi_{\nabla u}^{\prime}(\nabla u, z)=\hat{\varphi}_{F}^{\prime}(\mathbb{I}+\nabla u, z)$ with $\varphi_{\nabla u}^{\prime}$ and $\hat{\varphi}_{F}^{\prime}$ denoting the tensor-valued partial gradients.

If the displacement gradient $\nabla u$ is small, one can neglect the quadratic term (4.1) so that the GreenLagrange strain tensor $E$ from (4.6) turns into a so-called small-strain tensor $e(u):=\frac{1}{2} \nabla u+\frac{1}{2}(\nabla u)^{\top}$, i.e. $e_{i j}(u)=\frac{1}{2} \frac{\partial u_{i}}{\partial x_{j}}+\frac{1}{2} \frac{\partial u_{j}}{\partial x_{i}}, i, j=1, \ldots, 3$.

For all examples below, we assume $\Omega \subset \mathbb{R}^{3}$ to be a polyhedral domain. The discretization is made by a family of regular triangulations of $\Omega$ with the mesh parameter $h>0$ and $\Pi_{U, h}$ and $\Pi_{Z, h}$ will always be considered as quasi-interpolation operators related with standard conformal finite elements of polynomial type, namely $\mathrm{P} 0$ (i.e. element-wise constant functions) or $\mathrm{P} 1$ (i.e. element-wise affine continuous functions). To be more explicit, we can consider a mollifier $u \mapsto \tilde{u}_{h}$ with $\tilde{u}_{h}(x)=\int_{\Omega} k_{h}(x, \xi) u(\xi) \mathrm{d} \xi$ using a continuous kernel $k_{h}: \Omega \times \Omega \rightarrow \mathbb{R}^{+}$ supported on an $h$-neighborhood of the diagonal in $\Omega \times \Omega$ and $\int_{\Omega} k_{h}(x, \xi) \mathrm{d} \xi=1$ for all $x \in \Omega$. Then define $u_{h}=\Pi_{U, h} u$ as a Lagrange piecewise affine interpolation of $\tilde{u}_{h}$ using the nodal points in case of P1-elements, or piecewise constant interpolation using barycenters of the simplexes of the particular triangulation in case of $\mathrm{P} 0$-elements. Moreover, we will assume the triangulations conformal with the specific disjoint partition of $\Gamma$ where possibly different boundary conditions are prescribed. As to the initial condition $q_{0}$, we will always assume its stability (2.35), e.g. ensured by a "gentle start" (3.11) and thus not discussed in particular cases.

\subsection{Plasticity with hardening at small strains}

The first example on which we want to demonstrate our theory is a fully rate-independent plasticity with isotropic hardening. This is a standard model for inelastic materials for which existence, uniqueness and numerical convergence results are classical, see [22,61-63] for a survey. However, most of the numerical results derive convergence rates under the assumption of unjustified higher regularity, $c f$. the above references and e.g., [2]. Here we show that our abstract method provides an alternate proof of the result in [23], where strong convergence without rates was proved "under conditions of minimal regularity", namely those that follow directly from the existence theory.

The vector of the internal parameters $z:=(\pi, \eta) \in L^{2}\left(\Omega ; \mathbb{R}_{\mathrm{sym}, 0}^{3 \times 3}\right) \times L^{2}(\Omega)$ is therefore now composed from the plastic strain $\pi$ and a hardening variable $\eta$; here we used the notation

$$
\mathbb{R}_{\mathrm{sym}, 0}^{3 \times 3}:=\left\{A \in \mathbb{R}^{3 \times 3} ; \quad A^{\top}=A, \quad \operatorname{tr}(A)=0\right\} .
$$

For simplicity, we consider homogeneous Dirichlet boundary conditions on a part $\Gamma_{0}$ of the boundary $\partial \Omega$ with nonvanishing surface measure, so that

$$
\mathcal{U}:=U=\left\{u \in W^{1,2}\left(\Omega ; \mathbb{R}^{3}\right) ; u=0 \text { a.e. on } \Gamma_{0}\right\} \quad \text { and } \quad \mathcal{Z}:=L^{2}\left(\Omega ; \mathbb{R}_{\mathrm{sym}, 0}^{3 \times 3} \times \mathbb{R}\right) \cap K
$$

where $K$ is the cone of admissible evolution directions, see (4.11) below. We postulate the stored energy as

$$
\mathcal{E}(t, u, \pi, \eta):=\frac{1}{2} \int_{\Omega}(e(u)-\pi): \mathbb{C}:(e(u)-\pi)+b \eta^{2} \mathrm{~d} x-\langle f(t), u\rangle \quad \text { with }\langle f(t), u\rangle=\int_{\Gamma_{1}} g(t, x) \cdot u(x) \mathrm{d} S,
$$


where $\mathbb{C}=\left[\mathbb{C}_{i j k l}\right] \in \mathbb{R}^{3 \times 3 \times 3 \times 3}$ is a positive-definite 4 th-order tensor of elastic moduli, $\sigma: e=\sum_{i, j=1}^{3} \sigma_{i j} e_{i j}$, $b>0$ a hardening parameter, $\Gamma_{1}:=\partial \Omega \backslash \Gamma_{0}$, and $g \in C^{1}\left([0, T] ; L^{2}\left(\Gamma_{1}\right)\right)$.

The hardening is a unidirectional process and is, in standardly accepted models, reflected by the cone of admissible evolution directions in the form

$$
K:=\left\{(\pi, \eta) \in L^{2}\left(\Omega ; \mathbb{R}_{\mathrm{sym}, 0}^{3 \times 3} \times \mathbb{R}\right) ; \quad \delta_{P}^{*}(\pi) \leq \eta \text { a.e. on } \Omega\right\},
$$

where $P \subset \mathbb{R}_{\text {sym }, 0}^{3 \times 3}$ is closed, bounded, convex, and contains 0 in its interior and $\delta_{P}^{*}(\pi)=\max \{\pi: \sigma ; \sigma \in P\}$. The associated dissipation potential takes the form

$$
R(\dot{\pi}, \dot{\eta}):= \begin{cases}\int_{\Omega} \delta_{P}^{*}(\dot{\pi}) \mathrm{d} x & \text { if } \delta_{P}^{*}(\dot{\pi}) \leq \dot{\eta} \text { a.e. on } \Omega \\ +\infty & \text { otherwise. }\end{cases}
$$

This leads naturally to $Z_{1}:=L^{1}\left(\Omega ; \mathbb{R}_{\mathrm{sym}, 0}^{3 \times 3}\right) \times L^{1}(\Omega)$ in Remark 3.2. Beside the natural initial condition $\eta(0, \cdot)=1$, we must prescribe $\pi(0, \cdot)=\pi_{0} \in L^{2}\left(\Omega ; \mathbb{R}_{\mathrm{sym}, 0}^{3 \times 3}\right)$. The required stability $(2.35)$ of $q_{0}$, achieved e.g. through the "gentle start" (3.11) as suggested in Section 4.1, yields $z_{0}=\left(\pi_{0}, \eta_{0}\right) \in K$, i.e. here $\delta_{K}^{*}\left(\pi_{0}\right) \leq 1$. The mentioned initial condition $\eta_{0}=1$ is, in general, guaranteed by this way only if $f(0)$ is small enough. Moreover, it is well-known (cf. [22,37]) that this problem has a unique energetic solution $(u, z) \in W^{1, \infty}([0, T] ; U \times Z)$.

We assume a polyhedral domain $\Omega$ with also $\Gamma_{0}$ and $\Gamma_{1}$ having a polyhedral shape, and assume $\Omega$ triangulated by a family of regular triangulations with the mesh parameter $h>0$ conformal with the partition $\Gamma=\Gamma_{0} \cup \Gamma_{1}$, and $\Pi_{U, h}$ and $\Pi_{Z, h}$ quasi-interpolation operators related with conformal P1-elements and P0-elements, respectively. It is also important that the P0-elements are conformal with the cone $K$ from (4.11) used also for $\mathcal{Z}$ in (4.9) in the sense $\Pi_{Z, h} K \subset K$, as needed for Proposition 3.5. As there is no $\Xi$ in this problem, we have $\mathcal{E}_{\varepsilon}=\mathcal{E}$ but $R_{\varepsilon}$ from (3.9) is to be considered (unless one thinks about $R+\delta_{K}$ in place of $R_{\varepsilon}$ as suggested in Rem. 3.6), and also (3.25) with $\sigma$ the norm topology works simply for $\left[q_{0}\right]_{h, \varepsilon}:=\Pi_{h} q_{0}$.

Theorem 4.2. Let the data $\Omega, \Gamma_{0}, \Gamma_{1}, P$, and $q_{0}$ be qualified as above, and $g \in C^{1}\left([0, T] ; L^{4 / 3}\left(\Gamma_{1} ; \mathbb{R}^{3}\right)\right)$ and $\left[q_{0}\right]_{h, \varepsilon}$ be taken as above. Then the approximate solutions $q_{\varepsilon, \tau, h}=\left(u_{\varepsilon, \tau, h}, \pi_{\varepsilon, \tau, h}, \eta_{\varepsilon, \tau, h}\right)$ with

$$
\begin{aligned}
& u_{\varepsilon, \tau, h} \in L^{\infty}\left(0, T ; W^{1,2}\left(\Omega ; \mathbb{R}^{3}\right)\right), \\
& \pi_{\varepsilon, \tau, h} \in L^{\infty}\left(0, T ; L^{2}\left(\Omega ; \mathbb{R}_{\mathrm{sym}, 0}^{3 \times 3}\right)\right) \cap \operatorname{BV}\left([0, T] ; L^{1}\left(\Omega ; \mathbb{R}^{3 \times 3}\right)\right), \\
& \eta_{\varepsilon, \tau, h} \in L^{\infty}\left(0, T ; L^{2}(\Omega)\right) \cap \operatorname{BV}\left([0, T] ; L^{1}(\Omega)\right),
\end{aligned}
$$

based on the P0-elements for $\pi$ and $\eta$ and the P1-elements for $u$ converge, for all $t \in[0, T]$, strongly in $W^{1,2}\left(\Omega ; \mathbb{R}^{3}\right) \times L^{2}\left(\Omega ; \mathbb{R}_{\mathrm{sym}, 0}^{3 \times 3} \times \mathbb{R}\right)$ for $(\varepsilon, \tau, h) \rightarrow(0,0,0)$ to the energetic solution of the initial value problem given by $\left(\mathcal{U} \times \mathcal{Z}, \mathcal{E}, R, q_{0}\right)$.

The proof is a direct application of Proposition 3.5, since it is routine to check that its assumptions hold, cf. $[43,45]$. Here it is important to realize that the choice of $K$ and $R$ via $\delta_{P}^{*}$ is such that the coercivity $\mathcal{E}(t, u, \pi, \eta) \geq c\left(\|u\|_{W^{1,2}\left(\Omega ; \mathbb{R}^{3}\right)}^{2}+\|(\pi, \eta)\|_{L^{2}\left(\Omega ; \mathbb{R}^{3 \times 3} \times \mathbb{R}\right)}^{2}\right)-\|g\|_{L^{4 / 3}}\|u\|_{W^{1,2}\left(\Omega ; \mathbb{R}^{3}\right)}$ holds on $K$.

Note that the whole sequence converges since the limit solution is unique, $c f$. Remark 3.2. Moreover the convergence is strong by the uniform convexity of $\mathcal{E}$, see Remark 2.7 .

Remark 4.3 (implementation without regularization by LQ-program). In anisotropic media like single-crystals, the domain $P$ is considered to be polyhedral, $c f . e . g . ~[12]$, hence $\delta_{P}^{*}$ has a polyhedral epigraph and the incremental problem (2.22) without any regularization (cf. Rem. 3.6) represents a minimization problem of a sum of a quadratic and a polyhedral-graph functional which can be, after a computationally cheap enhancement, solved by efficient linear-quadratic solvers; $c f$. [55], Lemma 4, for this enhancement. 


\subsection{Phase transformation: a mixture approach}

In engineering, modeling of inelastic response of the materials undergoing martensitic transformation is of high interest. Here we demonstrate our theory on a simplified mixture-like model for martensitic transformation. Taking $\Gamma_{0}$ as in Section 4.2 and $Z_{0}:=\left\{s \in \mathbb{R}^{m} ; s_{l} \geq 0 \& \sum_{l=1}^{m} s_{l}=1\right\}$ the Gibbs simplex, we put

$$
\begin{aligned}
& \mathcal{U}:=U=\left\{u \in W^{1, p}\left(\Omega ; \mathbb{R}^{3}\right) ; \quad u=0 \text { a.e. on } \Gamma_{0}\right\} \\
& \mathcal{Z}:=\left\{z \in Z:=W^{\alpha, 2}\left(\Omega ; \mathbb{R}^{m}\right) ; \quad z(x) \in Z_{0} \text { for a.a. } x \in \Omega\right\}
\end{aligned}
$$

with $\alpha>0$ denoting (possibly a fractional) order of derivatives of the vector of the internal parameters $z$ which now represents volume fractions referring to $m$ phases (or phase variants). For simplicity, we consider the loading again through $g$ as in Section 4.2, i.e. $f$ is again defined by (4.10). We postulate the stored energy as

$$
E(u, z):=\int_{\Omega} \varphi(\nabla u, z) \mathrm{d} x+\frac{\kappa}{2}|z|_{\alpha}^{2}
$$

with $\kappa, \alpha>0$ and $|\cdot|_{\alpha}$ denoting the usual seminorm in the Sobolev (or, for $\alpha$ noninteger, Sobolev-Slobodetskiı) space, i.e.

$$
|z|_{\alpha}^{2}= \begin{cases}\int_{\Omega}\left|\nabla^{\alpha} z\right|^{2} \mathrm{~d} x & \text { for } \alpha \in \mathbb{N}, \\ \frac{1}{4} \int_{\Omega} \int_{\Omega} \frac{\left|\nabla^{[\alpha]} z(x)-\nabla^{[\alpha]} z(\xi)\right|^{2}}{|x-\xi|^{3+2(\alpha-[\alpha])} \mathrm{d} x \mathrm{~d} \xi} & \text { for } \alpha>0 \text { noninteger }\end{cases}
$$

with $[\alpha]$ the integer part of $\alpha$. In principle, more physically justified kernels with a support localized around the diagonal $\{x=\xi\}$ with the same singular behavior as $|x-\xi|^{-3-2(\alpha-[\alpha])}$ for $|x-\xi| \rightarrow 0$ could equally be used in (4.17).

The degree-1 homogeneous dissipation potential is now postulated as

$$
R(z):=\int_{\Omega} \delta_{M}^{*}(z) \mathrm{d} x
$$

where $\delta_{M}^{*}$ is determined, in analogy with $\delta_{P}^{*}$ from Section 4.2 , by a convex compact neighborhood $M \subset \mathbb{R}^{m}$ of the origin which prescribes activation energies for martensite/austenite phase-transformation or for re-orientation of particular martensitic variants. In particular, the martensitic transformation is a reversible process, so that $K=Z$. Also, there is nor $\Xi$ neither $K \neq Z$ and thus both $\mathcal{E}_{\varepsilon} \equiv \mathcal{E}$ and $\mathcal{D}_{\varepsilon} \equiv \mathcal{D}$ and the $\varepsilon$-regularization is irrelevant here.

For the discretization, we consider naturally P1-elements for $u$ and either P0-elements for $z$ (if $\alpha<1 / 2$ ) or P1-element also for $z$ if $(\alpha<3 / 2)$. Again, taking $\left[q_{0}\right]_{h, \varepsilon}:=\Pi_{h} q_{0}$ guarantees (3.25) with $\sigma$ being the norm topology.

Theorem 4.4. Let the data $\Omega, \Gamma_{0}$, and $\Gamma_{1}$ be qualified as in Section 4.2, let $\varphi$ be qualified as in Lemma 4.1 (now $p_{1}$ is irrelevant, as $Z_{0}$ is bounded here), let $M$ in (4.18) be a bounded convex neighbourhood of $0 \in \mathbb{R}^{m}$, let

$$
g \in C^{1}\left([0, T] ; L^{p^{\#} /\left(p^{\#}-1\right)}\left(\Gamma_{1} ; \mathbb{R}^{3}\right)\right), \quad \text { where } p^{\#} \begin{cases}=\frac{2 p}{3-p} & \text { for } p<3 \\ <+\infty & \text { for } p=3 \\ =+\infty & \text { for } p>3\end{cases}
$$

and let $q_{0} \in \mathcal{S}(0)$ be approximated by $\left[q_{0}\right]_{h, \varepsilon}:=\Pi_{h} q_{0}$. Then the approximate solutions $q_{\tau, h}=\left(u_{\tau, h}, z_{\tau, h}\right)$ with

$$
\begin{aligned}
& u_{\tau, h} \in L^{\infty}\left(0, T ; W^{1, p}\left(\Omega ; \mathbb{R}^{3}\right)\right), \\
& z_{\tau, h} \in L^{\infty}\left(0, T ; W^{\alpha, 2}\left(\Omega ; \mathbb{R}^{m}\right)\right) \cap \operatorname{BV}\left([0, T] ; L^{1}\left(\Omega ; \mathbb{R}^{m}\right)\right),
\end{aligned}
$$


based on the P1-elements for $u$ and the P0- or P1-elements for $z$ converge for $(\tau, h) \rightarrow(0,0)($ in terms of subsequences in the sense of Thm. 2.6 with Rem. 3.2) to energetic solutions of the problem given by $E, R, f$ and $q_{0}$ above.

Proof. Coercivity on $\mathcal{Q}=\mathcal{U} \times \mathcal{Z}$ follows from the assumed coercivity $(4.2)$ of $\varphi(\cdot, z)$ by Poincaré inequality combined with the Dirichlet condition on $\Gamma_{0}$ and by the regularizing $\kappa$-term in (4.16) combined with the constraint $z(x) \in Z_{0}$ involved in $\mathcal{Z}$ in (4.15).

The lower-semicontinuity of the first term in (4.16) needed for (2.12) follows by Lemma 4.1 with $p_{1}<+\infty$ arbitrary since $Z_{0}$ is now bounded. The continuity of $R: L^{1}(\Omega) \rightarrow \mathbb{R}$ follows from (in fact is equivalent to) the assumed boundedness of $M \subset \mathbb{R}^{m}$ used in (4.18).

The assumption in Proposition 3.4 are satisfied simply if $\sigma$ : equals the strong topology on $W^{1, p}\left(\Omega ; \mathbb{R}^{3}\right) \times$ $W^{\alpha, 2}\left(\Omega ; \mathbb{R}^{m}\right)$. Here the convexity of the Gibbs simplex $Z_{0}$ involved in $\mathcal{Z}$ is used, which makes both P0- and P1-elements compatible with $\mathcal{Z}$ in the sense $\Pi_{Z, h} \mathcal{Z} \subset \mathcal{Z}$, cf. (3.3), which makes our results from Section 3.3 working.

Example 4.5. At small strains, a popular model takes a "mixture" of quadratic energies in the form

$$
\varphi(\nabla u, z):=\sum_{\ell=1}^{m} \frac{z_{\ell}}{2}\left(e(u)-e_{\ell}\right): \mathbb{C}_{\ell}:\left(e(u)-e_{\ell}\right)+\psi(z) \text { where } e_{\ell}:=\frac{U_{\ell}^{\top}+U_{\ell}}{2}
$$

with the distortion matrices $U_{\ell}$ of particular pure phases (or phase variants). The setting here is related with the situation of martensitic transformation in a single-crystal and $z$ 's are volume fractions of the so-called austenite and of particular variants of martensite, e.g. $m=4$ or 7 for tetragonal or orthorhombic martensite, respectively. The function $\psi$ reflects the difference between chemical energies of austenite and martensite and also between pure phases and "mixtures". As $\varphi(\cdot, z)$ is now convex, it qualifies for Lemma 4.1 with $Z_{0}$ bounded. The philosophy of mixtures of austenite/martensite phases in so-called shape-memory alloys has been proposed by Frémond [15]; in rate-independent variant also presented in [16]. For its analysis and numerical implementation see $[10,11,17,24,46]$. Gradients of mesoscopical volume fractions (i.e. (4.16) with $\alpha=1$ has already been used in Frémond's model [16], p. 364, or [17], Formula (7.20). Another way for obtaining physically relevant mixture energies is the quasiconvexification under volume constrains, also called cross-quasiconvexification, see [44]. For models with equal elastic moduli we refer e.g. to $[5,6,8,19,20,28,46,68]$.

\subsection{Phase transformation: non-mixture approach}

The mixture approach in Section 4.3 is rather designed for phenomenological models of polycrystals but is too coarse for the description of complicated microstructures occurring in shape-memory-alloy single-crystals. A microscopical model has been developed in [33] (see also [34]) by restricting $z$ to be valued in vertices of the Gibbs' simplex, i.e. only pure phase(variant)s are allowed; then $\alpha<1 / 2$ should be taken in (4.16) or, as considered in [33,34], a BV-like term $\kappa|\nabla z|$. In this model, $z$ "switches" $\varphi$.

A different philosophy with presumably similar effects, pioneered by Falk [13], considers the vectorial "order parameter" $z$ related to the deformation gradient $\nabla u$ and particular shapes are then switched rather by $\nabla u$. Spinodal regions are then allowed instead of mixtures. The specific stored energy $\varphi$ now depends only on $\nabla u$ but need not be quasiconvex. For example, in [3,4,31,54,57], a multiwell potential $\hat{\varphi}$ (related with $\varphi$ by (4.7)) arises by the combination of St.Venant-Kirchhoff materials considered for each particular phase:

$$
\hat{f}(F):=\min _{\ell=1, \ldots, m}\left(\frac{1}{2}\left(U_{\ell}^{-\top} F^{\top} F U_{\ell}^{-1}-\mathbb{I}\right): \mathbb{C}_{\ell}:\left(U_{\ell}^{-\top} F^{\top} F U_{\ell}^{-1}-\mathbb{I}\right)+c_{\ell}\right),
$$


where $U_{\ell}$ are distortion matrices as in Example 4.5, $\mathbb{C}_{\ell}$ are elastic-moduli tensors, $c_{\ell}$ are some constants, and $U_{\ell}^{-\top}:=\left(U_{\ell}^{\top}\right)^{-1}$. Now naturally $p=4$. We postulate the stored energy in terms of $E$ and $\Xi$ as

$$
E(u, z):=\int_{\Omega} \varphi(\nabla u) \mathrm{d} x+\frac{\kappa}{2}|u|_{\alpha}^{2} \quad \text { and } \quad \Xi(u, z):=z-\mathcal{L}(\nabla u),
$$

with $\kappa>0, \alpha>1$ and $\mathcal{L}: \mathbb{R}^{3 \times 3} \rightarrow Z_{0}$ playing the role of a "phase indicator" with $Z_{0}$ being again the Gibbs simplex. The seminorm $|\cdot|_{\alpha}$ defined in (4.17) used for $1<\alpha<2$ with the Frobenius norm in the enumerator, now acting on $(3 \times 3)$-matrices is frame-indifferent, as observed by Arndt in [3]. We consider the same loading as in Sections 4.2 and 4.3, i.e. $f$ from (4.10), but now we put

$$
\begin{aligned}
& \mathcal{U}:=U=\left\{u \in W^{\alpha, 2}\left(\Omega ; \mathbb{R}^{3}\right) ; \quad u=0 \text { a.e. on } \Gamma_{0}\right\} \\
& \mathcal{Z}:=\left\{z \in Z:=L^{2}\left(\Omega ; \mathbb{R}^{m}\right) ; z(x) \in Z_{0} \text { for a.a. } x \in \Omega\right\},
\end{aligned}
$$

and then naturally $X:=Z$. The dissipation potential $R$ is again from (4.18). There is no $K$ involved, hence $\mathcal{D}_{\varepsilon}=\mathcal{D}$, but as $\Xi$ from (4.23) occurs, the regularization $\mathcal{E}_{\varepsilon}$ is, in principle, to be considered.

Choosing $\alpha<3 / 2$ allows for the usage of P1-elements for $u$ and P0-elements for $z$. As now $\mathcal{Q}=Q$ and $K=Z$, so in particular their conformity (3.3) is automatic. The proof of the following assertion shows that they are conformal also with the constraints $\Xi(q)=0$ so, in view of Remark 3.6, it would be possible to avoid the $\varepsilon$-regularization at all. When taking $\left[u_{0}\right]_{h, \varepsilon}=\Pi_{U, h} u_{0}$, we have $\nabla\left[u_{0}\right]_{h, \varepsilon}$ element-wise constant and so is $\mathcal{L}\left(\nabla\left[u_{0}\right]_{h, \varepsilon}\right)=:\left[z_{0}\right]_{h, \varepsilon}$, and $(3.25)$ is satisfied.

Theorem 4.6. Let $\varphi: \mathbb{R}^{3 \times 3} \rightarrow \mathbb{R}$ be continuous (not necessarily quasiconvex) satisfying (4.2) here with $m:=0$ (so no z-dependence), let $g$ satisfy (4.19), $\mathcal{L}: \mathbb{R}^{3 \times 3} \rightarrow Z_{0}$ be continuous, and $\alpha \in(1,3 / 2)$ and $p<6 /(5-2 \alpha)$ in $(4.2)$, and $q_{0}$ and $\left[q_{0}\right]_{h, \varepsilon}$ as specified above. Then the approximate solutions $q_{\varepsilon, \tau, h}=\left(u_{\varepsilon, \tau, h}, z_{\varepsilon, \tau, h}\right)$ with

$$
\begin{aligned}
& u_{\varepsilon, \tau, h} \in L^{\infty}\left(0, T ; W^{\alpha, 2}\left(\Omega ; \mathbb{R}^{3}\right)\right), \\
& z_{\varepsilon, \tau, h} \in L^{\infty}\left(0, T ; L^{\infty}\left(\Omega ; \mathbb{R}^{m}\right)\right) \cap \operatorname{BV}\left([0, T] ; L^{1}\left(\Omega ; \mathbb{R}^{m}\right)\right),
\end{aligned}
$$

based on the P1-elements for $u$ and the P0-elements for $z$ converge for $(\varepsilon, \tau, h) \rightarrow(0,0,0)$ (in terms of subsequences in the sense of Thm. 2.6 with Rem. 3.2) to energetic solutions of the problem given by $E, R, f$ and $q_{0}$ above.

Proof. Weak lower semicontinuity of $E$ is due to convexity of the regularizing term $\kappa|u|_{\alpha}^{2}$ in $(4.23)$ while $\varphi$ is now treated by compactness of the embedding $W^{\alpha, 2}(\Omega) \subset W^{1, p}(\Omega)$ (guaranteed if $p<6 /(5-2 \alpha)$ ) as a lowerorder term. The limit passage in the $z$-variable is trivial. This compactness also ensures the weak continuity of $\Xi: U \times Z \rightarrow X$.

As $K=Z$, condition (3.24) with $\sigma$ being the strong topology holds, if we show, for given $\tilde{z}=\mathcal{L}(\nabla \tilde{u})$, the existence of $\left(\tilde{u}_{h}, \tilde{z}_{h}\right) \stackrel{\sigma}{\rightarrow}(\tilde{u}, \tilde{z})$ such that $\tilde{z}_{h}=\mathcal{L}\left(\nabla \tilde{u}_{h}\right)$. As far as $\tilde{u}_{h}$, this can be done by a density argument of smooth functions in $W^{\alpha, 2}\left(\Omega ; \mathbb{R}^{3}\right)$, and then the usual Lagrange interpolation. By the embedding $W^{\alpha, 2}(\Omega) \subset W^{1, p}(\Omega), \nabla \tilde{u}_{h} \stackrel{\mathrm{s}}{\rightarrow} \nabla \tilde{u}$ in $L^{p}\left(\Omega ; \mathbb{R}^{3 \times 3}\right)$ and $\tilde{z}_{h}=\mathcal{L}\left(\nabla \tilde{u}_{h}\right) \stackrel{\mathrm{s}}{\rightarrow} \mathcal{L}(\nabla \tilde{u})=\tilde{z}$ by continuity of the Nemytskiı mapping induced by $\mathcal{L}$.

Then we use the results from Section 2.1 via Proposition 3.4 with $\sigma$ being the strong topology on $W^{\alpha, 2}\left(\Omega ; \mathbb{R}^{3}\right) \times$ $L^{2}\left(\Omega ; \mathbb{R}^{m}\right)$.

Remark 4.7. The inequalities $\alpha<3 / 2$ and $p<6 /(5-2 \alpha)$ restrict us to $p<3$, which unfortunately excludes (4.22). Hence we are tempted to take higher $\alpha$ which, however, brings the necessity to use higher-order elements (or to split the problem to a system). Considering P2-elements for $u$ would allow for $\alpha<5 / 2$ which, in turn, would allow for arbitrarily high $p$. Since $\mathcal{L}$ is inevitable nonlinear, it is no longer conformal with the constraint $\Xi(q)=0$ no matter what (polynomial) elements are taken for $z$. This would drive us to a penalization technique based on Proposition 3.3. However, here it is simpler to modify our analysis to allow for expressing the model in terms of $u$ only, $c f$. the following Remark 4.8 . 
Remark 4.8. In fact, a "viscous" rate-dependent variant of the above model was proposed in [54], for the rateindependent dissipation term $c f$. [54], Formula (33). The regularizing term $|\cdot|_{\alpha}$ used for $\alpha<1 / 2$ and the P0/P1discretization was suggested and implemented in [3] and computational experiments on NiMnGa single crystals reported in [4]. In [50], the model was analyzed and implemented in the 1-dimensional case with $\alpha=2$. Pure analysis then followed also in [51]; in particular for $\alpha \geq 3$, [51], Proposition 3, investigated an inviscid variant of this model accounting, contrary to our paper, also for inertial effects. In fact, the model was formulated only in terms of $u$ in $[3,50,51,54]$ but then the dissipation distance took the form $\mathcal{D}\left(u_{1}, u_{2}\right)=\int_{\Omega}\left|\mathcal{L}\left(\nabla u_{1}\right)-\mathcal{L}\left(\nabla u_{2}\right)\right| \mathrm{d} x$, having lost the structure based on the degree-1 homogeneous potential $R$. Neglecting difficulties in numerical evaluation of such $\mathcal{D}$ if $\alpha=2$ would be considered, by this way one gets rid of the necessity to penalize $\Xi$ (which, in case $\alpha<1 / 2$, is made possible due to Thm. 4.6 together with Rem. 3.6 in our case too). Nevertheless, a fully rate-independent model, used in fact for calculations in [4], has not been subjected to any rigorous mathematical/numerical analysis, and therefore Theorem 4.6 brings indeed new results.

\subsection{Damage at large strains}

In engineering, other inelastic process in the materials of a high interest is damage. We consider a fully rateindependent isotropic and nonlocal damage, and again consider the body $\Omega$ fixed on a nonvanishing part $\Gamma_{0}$ and loaded by a surface force $g$ on $\Gamma_{1}=\partial \Omega \backslash \Gamma_{0}$, so that $\mathcal{U}=U$ is again from (4.14). As we consider isotropic damage, the internal parameter $z \in \mathcal{Z}$ will be scalar valued with

$$
\mathcal{Z}:=\left\{z \in Z:=W^{\alpha, 2}(\Omega) ; \quad z(x) \geq 0 \text { for a.a. } x \in \Omega\right\}
$$

We postulate the stored energy again by the formula $(4.16) ; \kappa>0$ in (4.16) is now a coefficient responsible for nonlocal effects in gradient-of-damage theories as, e.g., in [16], cf. [40] for a discussion and more references. Note that we admitted, rather formally, $\varphi$ operating on the argument $z$ nonrestricted from above to allow for a simple construction of the recovery sequence (3.31). The loading $f$ is considered again by (4.10).

Like isotropic hardening in Section 4.2, the process of damaging is unidirectional in the sense that, if in progress, it can only increase but the material never can heal, which leads us to define the cone of admissible evolution directions and the degree-1 homogeneous dissipation potential as

$$
K:=\left\{z \in W^{\alpha, 2}(\Omega) ; z \geq 0 \text { a.e. on } \Omega\right\} \equiv \mathcal{Z} \quad \text { and } \quad R(z):=\int_{\Omega} c_{1} z \mathrm{~d} x
$$

where $c_{1}$ is a phenomenological specific energy (with physical dimension $\mathrm{J} \cdot \mathrm{m}^{-3}=\mathrm{Pa}$ ) expressing the energy needed for a damage of a unit volume described by a unit jump of the damage parameter $z$. Considering the initial condition for $z_{0}=0$ and $\varphi(A, \cdot)$ decreasing for $z \in[0,1]$ and with $\varphi(A, z)=\varphi(A, 1)+(z-1)^{2}$ for $z \in(1,+\infty)$, we effectively force the values of $z$ to range only the interval $[0,1]$ and $c_{1}$ refers to the specific energy dissipated by damaging the original material (having the stored-energy $\varphi(\cdot, 0)$ ) to the fully damaged material (having the stored-energy $\varphi(\cdot, 1)$ assumed to be still coercive so we exclude the case when the material fully disintegrates).

As no equality constraints of the type $\Xi(q)=0$ are involved, we have $\mathcal{E}_{\varepsilon}=\mathcal{E}$ but the $\varepsilon$-regularization $\mathcal{D}_{\varepsilon}$ from (3.9) is to be still considered unless one takes $R+\delta_{K}$ instead of $R_{\varepsilon}$, cf. Remark 3.6. For the discretization, as in Section 4.3, we consider P1-elements for $u$ and either P0-elements for $z$ (if $\alpha<1 / 2$ ) or P1-element also for $z$ if $(\alpha<3 / 2)$. Again, both P0- and P1-elements are conformal with the constraints in $\mathcal{Z}=K$ from (4.27)-(4.28) in the sense $\Pi_{Z, h} \mathcal{Z} \subset \mathcal{Z}$ and $\Pi_{Z, h} K \subset K$, as required in Proposition 3.5.

Theorem 4.9. Let the data $\Omega, \Gamma_{0}$, and $\Gamma_{1}$ be qualified as in Section 4.2 , let $\varphi$ be qualified as in Lemma 4.1 with $m:=1$ and $Z_{0}:=\{z \geq 0\}$ and $p_{1}:=2$, let $g$ satisfy (4.19), and let $q_{0} \in \mathcal{S}(0)$ and $\left[q_{0}\right]_{h, \varepsilon}:=\Pi_{h} q_{0}$. 
Then the approximate solutions $q_{\varepsilon, \tau, h}=\left(u_{\varepsilon, \tau, h}, z_{\varepsilon, \tau, h}\right)$ with

$$
\begin{aligned}
& u_{\varepsilon, \tau, h} \in L^{\infty}\left(0, T ; W^{1, p}\left(\Omega ; \mathbb{R}^{3}\right)\right), \\
& z_{\varepsilon, \tau, h} \in L^{\infty}\left(0, T ; W^{\alpha, 2}(\Omega)\right) \cap \operatorname{BV}\left([0, T] ; L^{1}(\Omega)\right),
\end{aligned}
$$

based on the P1-elements for $u$ and the P0- or P1-elements for $z$ converge for $(\varepsilon, \tau, h) \rightarrow(0,0,0)$ (in terms of subsequences in the sense of Thm. 2.6 with Rem. 3.2) to energetic solutions of the problem given by $E, R, K$, $f$ and $q_{0}$ above.

Proof. Coercivity on $\mathcal{Q}=\mathcal{U} \times \mathcal{Z}$ follows from the assumed condition $|A|^{p} \leq \varphi(A, z)$ by the Poincaré inequality combined with the Dirichlet condition on $\Gamma_{0}$ and by the regularizing $\kappa$-term in (4.16) combined with the constraint $z(x) \geq 0$ involved in $\mathcal{Z}$ in (4.27). Then we use Proposition 3.5 with the decomposition (3.30) using $B=E_{1}^{\prime}$ with $E_{1}(z):=\frac{\kappa}{2}|z|_{\alpha}^{2}$ and $E_{0}(u, z)=\int_{\Omega} \varphi(\nabla u, z) \mathrm{d} x$. Note also that $\left[q_{0}\right]_{h, \varepsilon}:=\Pi_{h} q_{0}$ satisfies (3.25).

Remark 4.10. The partial damage at large strains has been analyzed in [40] but without any numerical approximation and a nonquadratic regularizing term $\frac{\kappa}{p_{1}}|\nabla z|^{p_{1}}$ with $p_{1}>3$ had to be used, contrary to the quadratic term in (4.16) which is usual in engineering literature but never was mathematically analyzed so far. Hence Theorem 4.9 represents a new extension in this field.

\subsection{Debonding at large strains}

Other inelastic processes may occur not in the materials themselves but on the boundary. Here we want to consider a possible debonding of an elastic support on a part $\Gamma_{2}$ of the boundary $\partial \Omega$. The internal parameter $z \in L^{\infty}\left(\Gamma_{2}\right)$ is therefore now a scalar debonding parameter assumed to range [0,1] and expressing volume fraction of the adhesive which fixes elastically the body on $\Gamma_{2}$ if not debonded. It is natural also to consider a unilateral Signorini contact on $\Gamma_{2}$. Moreover, we again consider the body $\Omega$ fixed on a nonvanishing part $\Gamma_{0}$ of $\partial \Omega$ (disjoint with $\left.\Gamma_{2}\right)$ and loaded by a surface time-varying force $g$ on $\Gamma_{1}=\partial \Omega \backslash\left(\Gamma_{0} \cup \Gamma_{2}\right)$, so that

$$
\begin{aligned}
& \mathcal{U}:=\left\{u \in W^{1, p}\left(\Omega ; \mathbb{R}^{3}\right) ; \quad u=0 \text { a.e. on } \Gamma_{0}, \quad \nu \cdot u \geq 0 \text { a.e. on } \Gamma_{2}\right\} \\
& \mathcal{Z}:=\left\{z \in Z:=L^{\infty}\left(\Gamma_{2}\right) ; \quad 0 \leq z \leq 1 \text { a.e. on } \Gamma_{2}\right\}
\end{aligned}
$$

with $\nu=\nu(x)$ a normal to $\Gamma_{2}$. We postulate the stored energy as

$$
E(u, z):=\int_{\Omega} \varphi(\nabla u) \mathrm{d} x+\int_{\Gamma_{2}}(1-z) \psi(u) \mathrm{d} S
$$

where $\psi: \mathbb{R}^{3} \rightarrow \mathbb{R}_{+}$describes the elastic response of the adhesive fixing the body on $\Gamma_{2}$.

Considering naturally that debonding can only develop but never heal back leads us to pose the cone of admissible evolution directions and, similarly like in (4.28), the degree-1 homogeneous dissipation potential as

$$
K:=\left\{z \in L^{\infty}\left(\Gamma_{2}\right) ; z \geq 0 \text { a.e. on } \Gamma_{2}\right\} \quad \text { and } \quad R(z):=\int_{\Gamma_{2}} c_{2} z \mathrm{~d} S
$$

with $c_{2}$ a phenomenological specific energy (with physical dimension $\mathrm{J} \cdot \mathrm{m}^{-2}$ ) expressing the energy needed for a full debonding of a unit area of $\Gamma_{2}$.

Natural finite-element approximation is now P1-elements for $u$ and P0-elements on the boundary for $z$. We assume that the disjoint partition $\Gamma=\Gamma_{0} \cup \Gamma_{1} \cup \Gamma_{2}$ is polyhedral and that the triangulations are conformal with this partition. To simplify technical details, let us assume that $\Gamma_{2}$ is flat; this ensures $\Pi_{U, h} \mathcal{U} \subset \mathcal{U}, c f$. (3.3). Also the constraints in (4.31) are conformal with $\mathrm{P} 0$-elements in the sense $\Pi_{Z, h} \mathcal{Z} \subset \mathcal{Z}$. As there is no $\Xi$ here, we have $\mathcal{E}_{\varepsilon} \equiv \mathcal{E}$ but $\mathcal{D}_{\varepsilon} \neq \mathcal{D}$ is still to be considered. 
Theorem 4.11. Let the disjoint partition $\Gamma=\Gamma_{0} \cup \Gamma_{1} \cup \Gamma_{2}$ be polyhedral, $\Gamma_{2}$ flat, and the triangulations be conformal with this partition, $\varphi$ be qualified as in Lemma 4.1 with $m:=0$ (i.e. with no $z$-dependence in $\varphi$ ), $g$ satisfy (4.19), and $\psi: \mathbb{R}^{3} \rightarrow \mathbb{R}$ be continuous satisfying the growth condition $0 \leq \psi(u) \leq C\left(1+|u|^{p^{\#}-\epsilon}\right)$ with $p^{\#}$ from (4.19) and $\epsilon>0$, and $q_{0} \in \mathcal{S}(0)$ is approximated by $\left[q_{0}\right]_{h, \varepsilon}:=\Pi_{h} q_{0}$. Then the approximate solutions $q_{\varepsilon, \tau, h}=\left(u_{\varepsilon, \tau, h}, z_{\varepsilon, \tau, h}\right)$ with

$$
\begin{aligned}
& u_{\varepsilon, \tau, h} \in L^{\infty}\left(0, T ; W^{1, p}\left(\Omega ; \mathbb{R}^{3}\right)\right), \\
& z_{\varepsilon, \tau, h} \in L^{\infty}\left(0, T ; L^{\infty}\left(\Gamma_{2}\right)\right) \cap \operatorname{BV}\left([0, T] ; L^{1}\left(\Gamma_{2}\right)\right),
\end{aligned}
$$

based on the P1-elements for $u$ and the P0-elements for $z$ converge for $(\varepsilon, \tau, h) \rightarrow(0,0,0)$ (in terms of subsequences in the sense of Thm. 2.6 with Rem. 3.2) to some energetic solutions of the problem given by $E, R, K$, $f$ and $q_{0}$ above.

Proof. The coercivity of $E$ follows as in Theorem 4.4; note that the term on $\Gamma_{2}$, being nonnegative, cannot destroy it. The weak lower-semicontinuity is again as in Theorem 4.4, the term on $\Gamma_{2}$ being even weakly continuous due to affinity in $z$-variable and due to the compactness of the trace operator $\left.u \mapsto u\right|_{\Gamma_{2}}: W^{1, p}\left(\Omega ; \mathbb{R}^{3}\right) \rightarrow L^{p^{\#}-\epsilon}\left(\Gamma_{2} ; \mathbb{R}^{3}\right)$.

We will explicitly construct the recovery sequence $\left\{\tilde{q}_{h}\right\}_{h>0}$ for (3.24). As to $\tilde{u}_{h}$ we use the construction (3.31a); as $\Gamma_{2}$ is flat, $\nu$ is constant on $\Gamma_{2}$, and $\Pi_{U, h} \mathcal{U}=U_{h} \cap \mathcal{U}$, which ensures $\mathcal{U}_{h} \subset \mathcal{U}$ because $\mathcal{U}_{h}:=\Pi_{U, h} \mathcal{U}$. As for $\Pi_{Z, h}$, we have in mind the standard Clément's quasi-interpolation by element-wise constant averages, hence e.g. functions valued in $[0,1]$ are again mapped to (element-wise constant) functions valued in $[0,1]$. We put

$$
\tilde{z}_{h}:=1-\left(1-z_{h}\right) \Pi_{Z, h}\left(\frac{1-\tilde{z}}{1-z}\right)
$$

If $z(x)=1$, then also $\tilde{z}(x)=1$ because always $z \leq \tilde{z} \leq 1$ and the fraction in (4.35) can be defined arbitrarily in valued $[0,1]$. The product of element-wise constant functions $1-z_{h}$ and $\Pi_{Z, h}\left(\frac{1-\tilde{z}}{1-z}\right)$ is again element-wise constant, hence $z_{h} \in Z_{h}$. As $0 \leq \Pi_{Z, h}\left(\frac{1-\tilde{z}}{1-z}\right) \leq 1$, we have also $z_{h} \leq \tilde{z}_{h} \leq 1$, hence $\tilde{z}_{h} \in \mathcal{Z}_{h}$ and $\tilde{z}_{h}-z_{h} \in K$. As $\Pi_{Z, h}\left(\frac{1-\tilde{z}}{1-z}\right) \stackrel{\mathrm{s}}{\rightarrow} \frac{1-\tilde{z}}{1-z}$ in any $L^{p}\left(\Gamma_{2}\right), p<+\infty$, and $z_{h} \stackrel{\mathrm{w}^{*}}{\rightarrow} z$, from $(4.35)$ we have $\tilde{z}_{h} \stackrel{\mathrm{w}^{*}}{\longrightarrow} 1-(1-z) \frac{1-\tilde{z}}{1-z}=\tilde{z}$ in fact in $L^{\infty}\left(\Gamma_{2}\right)$ due to the a priori bound of values in $[0,1]$.

Then, having (3.24) proved, we can verify (2.19) through Proposition 3.4 used with the topology $\sigma:=\mathrm{s} \times \mathrm{w}^{*}$ on $W^{1, p}\left(\Omega ; \mathbb{R}^{3}\right) \times L^{\infty}\left(\Gamma_{2}\right)$.

Remark 4.12. As we do not have any gradient-type regularization like in Section 4.5, we had to assume $E(u, \cdot)$ affine to allow for a passage via weak convergence. It however does not allow for any artificial definition like we did for $\varphi$ in Section 4.5 for $z>1$, which is why here we had to include the constraint $z(x) \in[0,1]$ explicitly into $\mathcal{Z}$ in (4.31) but this, in turn, destroyed any quadratic structure in $z$ and hence we had to rely on Proposition 3.4 supported by the rather sophisticated construction (4.35).

Remark 4.13. A debonding on a priori prescribed surfaces inside the body, called then rather a delamination, could be treated similarly only by introducing a more extensive notation, $c f$. [30]. Let us emphasize that Theorem 4.11 adapted to such a problem substantially improves results from [30], where convergence has only been proved for a semidiscretization in time while the convergence of the full time-space discretization has only silently been assumed under an additional convergence criterion $h / \tau \rightarrow 0$ and small strains.

\subsection{Magnetostriction at small strains}

In this section, we illustrate our theory on a deformable ferromagnet occupying a domain $\Omega \subset \mathbb{R}^{3}$ and undergoing quasistatic isothermal evolution at small strains. Again, the non-dissipative component $u: \Omega \rightarrow \mathbb{R}^{3}$ will be the displacement while the dissipating variable $z: \Omega \rightarrow \mathbb{R}^{3}$ will now be the magnetization vector; 
thus $m=3$ here. The stored energy is then considered in the form

$$
E(u, z):=\int_{\Omega}\left(\varphi(\nabla u(x), z(x))+\frac{\kappa}{2}|\nabla z|^{2}\right) \mathrm{d} x+\frac{\mu_{0}}{2} \int_{\mathbb{R}^{3}}|\nabla \phi|^{2} \mathrm{~d} x .
$$

The particular terms in (4.36) represent the mechanical stored energy interacting with an anisotropic magnetization energy, the exchange energy (with $\kappa>0$ a coefficient having a quantum-mechanical origin), and the energy of the demagnetizing field $\phi \in W^{1,2}(\Omega)$ (with $\mu_{0}>0$ the vacuum permeability) which is determined by the magnetization $z$ by the (weak solution to the) following 2 nd-order linear elliptic equation on the whole space $\mathbb{R}^{3}$ :

$$
\operatorname{div}\left(\mu_{0} \nabla \phi-\chi_{\Omega} z\right)=0 \text { on } \mathbb{R}^{3},
$$

where $\chi_{\Omega}: \mathbb{R}^{3} \rightarrow\{0,1\}$ denotes the characteristic function on $\Omega$. The external forcing might be both mechanical and magnetic. Let us consider it again via a surface force $g$ (as in Sect. 4.2) and by an external magnetic field $h_{\text {ext }}$ :

$$
\langle f(t),(u, z)\rangle:=\int_{\Gamma_{1}} g(t, x) \cdot u(x) \mathrm{d} S+\int_{\Omega} h_{\text {ext }}(t, x) \cdot z(x) \mathrm{d} x .
$$

Contrary to the previous sections, $z$ is not any internal parameter because it can be subjected directly to outer loading by $h_{\text {ext }}$. For notational simplicity, we consider again the Dirichlet condition on $\Gamma_{0}$ and then take $\mathcal{U}=U$ from (4.14) while $Z$ is naturally to be taken as $W^{1,2}\left(\Omega ; \mathbb{R}^{3}\right)$. The standard model involves also the so-called Heisenberg constraint

$$
|z(x)|=m_{\mathrm{s}} \quad \text { for a.a. } x
$$

with $m_{\mathrm{s}}>0$ a given saturation magnetization. In fact, due to (4.39) we can redefine $\varphi(A, z)$ for $|z|>m_{\mathrm{s}}$, if needed, suitably so that the coercivity (4.2) holds. For the dissipation potential $R$ we consider, for example,

$$
R(z):=\int_{\Omega} d_{0}|z|+d_{1}\left|e_{3} \cdot z\right| \mathrm{d} x
$$

where $d_{0}>0$ and $d_{1} \geq 0$ and $e_{3}=(0,0,1)$. The $d_{0}$-term has been considered in [66] while for the $d_{1}$-term see [67] or also [55,56]. The former term corresponds to a basic dissipation and ensures coercivity of $R$ while the latter term describes dissipation during remagnetization in a uniaxial magnet with easy-magnetization axis in the direction $e_{3}$; then the anisotropic energy $\varphi(A, \cdot)$ is assumed to have minima along this axis and $d_{0}+d_{1}$ is a so-called coercive force which determines the width of a parent hysteresis loop during cyclic magnetization processes. The magnetization process is fully reversible (because we do not consider any sort of unidirectional "hardening" like in [56]) and therefore we put $K=Z=W^{1,2}\left(\Omega ; \mathbb{R}^{3}\right)$. The initial magnetization $z_{0}$ should satisfy the constraint (4.39) and, together with $u_{0}$, be stable with respect to the loading $h_{\text {ext }}(0, \cdot)$ and $g(0, \cdot)$; we will not specify this rather technical condition.

We cannot simply involve the constraint (4.39) into $\mathcal{Z}$ because (3.3) cannot conventionally be achieved because no polynomial finite elements are compatible with the Heisenberg constraints (4.39). Hence we implement it by $\Xi$ and then take simply $\mathcal{Z}:=Z=W^{1,2}\left(\Omega ; \mathbb{R}^{3}\right)$ and define $\Xi$ as

$$
\Xi: U \times Z \rightarrow X:=L^{2}(\Omega):(u, z) \mapsto \frac{|z|^{2}-m_{\mathrm{s}}^{2}}{\sqrt{|z|^{2}+m_{\mathrm{s}}^{2}}} .
$$

Note that the nonlinearity $r \mapsto\left(|r|^{2}-m_{\mathrm{s}}^{2}\right) / \sqrt{|r|^{2}+m_{\mathrm{s}}^{2}}$ involved in (4.41) has a linear growth and is Lipschitz continuous, and so is $\Xi: L^{2}\left(\Omega ; \mathbb{R}^{3} \times \mathbb{R}^{3}\right) \rightarrow L^{2}(\Omega)$. Simultaneously, $\Xi$ is weakly continuous on $U \times Z$ due to the compact embedding of $U \times Z$ into $L^{2}\left(\Omega ; \mathbb{R}^{3} \times \mathbb{R}^{3}\right)$. 
Again we consider a polyhedral domain $\Omega$ and its regular triangulations, and in view of (4.36) take P1elements both for $u$ and $z$. Then, in principle, exact integration formulae can be exploited for (4.37) and for the last term in (4.36), too. So no discretization of $\varphi$ would be needed, although practical calculations usually exploit some numerical approximation of this procedure (and hence of $E$ itself, too). As we did not consider it in previous sections, we omit it here too. Because of the mentioned incompatibility of the P1-elements (and in fact with any polynomial finite-elements), with the constraint $\Xi(u, z)=0$, i.e. $|z|=m_{\mathrm{s}}$, we must consider the penalization method. Using $\alpha=2$ in (3.5), it yields

$$
\mathcal{E}_{\varepsilon}(u, z)=\int_{\Omega}\left(\varphi(\nabla u(x), z(x))+\frac{\kappa}{2}|\nabla z|^{2}+\frac{\left(|z|^{2}-m_{\mathrm{s}}^{2}\right)^{2}}{\varepsilon\left(|z|^{2}+m_{\mathrm{s}}^{2}\right)}\right) \mathrm{d} x+\frac{\mu_{0}}{2} \int_{\mathbb{R}^{3}}|\nabla \phi|^{2} \mathrm{~d} x .
$$

The conformity (3.3) is here automatic because there are no other constraints involved, i.e. $\mathcal{Q} \equiv Q$ and $K \equiv Z$.

Theorem 4.14. Let the data $\Omega, \Gamma_{0}$, and $\Gamma_{1}$ be qualified as in Section 4.2 , let $\varphi$ be qualified as in Lemma 4.1 with $Z_{0}:=\mathbb{R}^{m}, m=3$, $p_{1}=2$, let $g$ satisfy (4.19), and let further $h_{\mathrm{ext}} \in C^{1}\left([0, T] ; L^{6 / 5}\left(\Omega ; \mathbb{R}^{3}\right)\right), q_{0} \in \mathcal{S}(0)$ and $\left[q_{0}\right]_{h, \varepsilon}:=\Pi_{h} q_{0}$. Then the approximate solutions $q_{\varepsilon, \tau, h}=\left(u_{\varepsilon, \tau, h}, z_{\varepsilon, \tau, h}\right)$ with

$$
\begin{aligned}
& u_{\varepsilon, \tau, h} \in L^{\infty}\left(0, T ; W^{1, p}\left(\Omega ; \mathbb{R}^{3}\right)\right), \\
& z_{\varepsilon, \tau, h} \in L^{\infty}\left(0, T ; W^{1,2}\left(\Omega ; \mathbb{R}^{3}\right)\right) \cap \operatorname{BV}\left([0, T] ; L^{1}\left(\Omega ; \mathbb{R}^{3}\right)\right),
\end{aligned}
$$

based on the P1-elements and the penalization of the Heisenberg constraint (4.39) as in (4.42) converge for $(\varepsilon, \tau, h) \rightarrow(0,0,0)$ (in terms of subsequences in the sense of Thm. 2.6 with Rem. 3.2) to energetic solutions of the problem given by $E, R, \Xi, f$ and $q_{0}$ above under the convergence criterion $h^{2} / \varepsilon \rightarrow 0$.

For the convergence criterion $h \leq H(\varepsilon)$ can take $H$, e.g., in the form

$$
H(\varepsilon)=\varepsilon^{a} \quad \text { with any } 0<a<1 / 2 .
$$

Proof of Theorem 4.14. The weak lower semicontinuity in the sense (2.12) of the $\varphi$-term in (4.42) is by Lemma 4.1, while that of the terms $|\nabla z|^{2}$ and $|\nabla \phi|^{2}$ is due to the convexity and linearity of (4.37). The penalty term in (4.42) has the 2nd-order-polynomial growth and is therefore continuous because of the compact embedding of $W^{1,2}(\Omega)$ into $L^{2}(\Omega)$. The coercivity of $\mathcal{E}$ on $U \times Z$ follows from (4.2) through Poincaré's inequality.

For our P1-elements, the estimate (3.19) with $\gamma=1$ is then known to hold with $|\cdot|$ and $\|\cdot\|$ being respectively the $L^{2}$ - and the $W^{1,2}$-norms. The Lipschitz continuity (3.18) of $\Xi$ from (4.41) holds for $X:=L^{2}(\Omega)$, which just makes the penalty form in (4.42) with $\alpha=2$. The choice $\left[q_{0}\right]_{h, \varepsilon}:=\Pi_{h} q_{0}$ again satisfies (3.25). Our assertion then follows from Theorem 2.6 through Proposition 3.3 where (3.20) just says that $h=o(\sqrt{\varepsilon})$, as claimed.

Remark 4.15. References for magnetostriction usually addresses large strains where more complications arise, cf. $[9,26,27,59,64]$. Mathematical analysis at large strains needs some additional regularization, e.g. like [59]. A conventional form of $\varphi$ in (4.36) in term of small strains, as considered here, is $\varphi(\nabla u, z)=\varphi_{0}(z)+\frac{1}{2}(e(u)-$ $\left.e_{z}\right): \mathbb{C}:\left(e(u)-e_{z}\right)$ with $e_{z}$ a preferred strain tensor corresponding to the magnetization $z$; for the concrete form of $e_{z}$ we refer to $[27,64]$. No numerical and even purely theoretical analysis of this rate-independent evolution problem seems to be reported in literature hence Theorem 4.14 represents a new result for this conceptual algorithm.

\section{REFERENCES}

[1] E. Acerbi and and N. Fusco, Semicontinuity problems in the calculus of variations. Arch. Ration. Mech. Anal. 86 (1984) $125-145$.

[2] J. Alberty and C. Carstensen, Numerical analysis of time-dependent primal elastoplasticity with hardening. SIAM J. Numer. Anal. 37 (2000) 1271-1294.

[3] M. Arndt, M. Griebel and T. Roubíček, Modelling and numerical simulation of martensitic transformation in shape memory alloys. Continuum Mech. Thermodyn. 15 (2003) 463-485. 
[4] M. Arndt, M. Griebel, V. Novák, T. Roubíček and P. Šittner, Martensitic transformation in NiMnGa single crystals: numerical simulations and experiments. Int. J. Plasticity 22 (2006) 1943-1961.

[5] F. Auricchio and L. Petrini, Improvements and algorithmical considerations on a recent three-dimensional model describing stress-induced solid phase transformations. Int. J. Numer. Meth. Engng. 55 (2002) 1255-1284.

[6] F. Auricchio, A. Mielke and U. Stefanelli, A rate-independent model for the isothermal quasi-static evolution of shape-memory materials. Math. Models Methods Appl. Sci. 18 (2008) 125-164.

[7] B. Bourdin, G. Francfort and J.-J. Marigo, Numerical experiments in revisited brittle fracture. J. Mech. Phys. Solids 48 (2000) $797-826$.

[8] J.G. Boyd and D.C. Lagoudas, A thermodynamical constitutive model for shape memory materials, Part I. The monolithic shape memory alloys. Int. J. Plasticity 12 (1996) 805-842.

[9] W.F. Brown, Magnetoelastic interactions, in Springer Tracts in Natural Philosophy 9, C. Truesdel Ed., Springer (1966).

[10] P. Colli and J. Sprekels, Global existence for a three-dimensional model for the thermo-mechanical evolution of shape memory alloys. Nonlinear Anal. 18 (1992) 873-888.

[11] P. Colli, M. Frémond and A. Visintin, Thermo-mechanical evolution of shape memory alloys. Quarterly Appl. Math. 48 (1990) $31-47$.

[12] S. Conti and M. Ortiz, Dislocation microstructures and effective behaviour of single crystals. Arch. Ration. Mech. Anal. 176 (2005) 103-147.

[13] F. Falk, Model free energy, mechanics and thermodynamics of shape memory alloys. Acta Metall. 28 (1980) $1773-1780$.

[14] G. Francfort and A. Mielke, An existence result for a rate-independent material model in the case of nonconvex energies. $J$. Reine Angew. Math. 595 (2006) 55-91.

[15] M. Frémond, Matériaux à mémoire deforme. C.R. Acad. Sci. Paris Sér. II 304 (1987) 239-244.

[16] M. Frémond, Non-Smooth Thermomechanics. Springer, Berlin (2002).

[17] E. Fried and M.E. Gurtin, Dynamic solid-solid transitions with phase characterized by an order perameter. Physica D 72 (1994) 287-308.

[18] A. Giacomini and M. Ponsiglione, Discontinuous finite element approximation of quasistatic crack growth in nonlinear elasticity. Math. Models Methods Appl. Sci. 16 (2006) 77-118.

[19] S. Govindjee and Ch. Miehe, A multi-variant martensitic phase transformation model: formulation and numerical implementation. Comp. Meth. Appl. Mech. Engr. 191 (2001) 215-238.

[20] S. Govindjee, A. Mielke and G.J. Hall, Free-energy of miixng for $n$-variant martensitic phase transformations using quasi-convex analysis. J. Mech. Phys. Solids 50 (2002) 1897-1922.

[21] B. Halphen and Q.S. Nguyen, Sur les matériaux standards généralisés. J. Mécanique 14 (1975) 39-63.

[22] W. Han and B.D. Reddy, Plasticity. Mathematical theory and numerical analysis. Springer, New York (1999).

[23] W. Han and B.D. Reddy, Convergence of approximations to the primal problem in plasticity under conditions of minimal regularity. Numer. Math. 87 (2000) 283-315.

[24] K.-H. Hoffmann, M. Niezgódka and Z. Songmu, Existence and uniqueness of global solutions to an extended model of the dynamical development in shape memory alloys. Nonlinear Anal. Theory Methods Appl. 15 (1990) 977-990.

[25] J.E. Huber, N.A. Fleck, C.M. Landis and R.M. McMeeking, A constitutive model for ferroelectric polycrystals. J. Mech. Phys. Solids 47 (1999) 1663-1697.

[26] R.D. James and D. Kinderlehrer, Theory of magnetostriction with applications to $\mathrm{Tb}_{x} \mathrm{Dy}_{1-x} \mathrm{Fe}_{2}$. Phil. Mag. 68 (1993) $237-274$.

[27] R.D. James and M. Wuttig, Magnetostriction of martensite. Phil. Mag. A 77 (1998) 1273.

[28] Y. Jung, P. Papadopoulos and R.O. Ritchie, Constitutive modeling and numerical simulation of multivariant phase transformation in superelastic shape-memory alloys. Int. J. Numer. Meth. Engng. 60 (2004) 429-460.

[29] D. Kinderlehrer and P. Pedregal, Gradient Young measures generated by sequences in Sobolev spaces. J. Geom. Anal. 4 (1994) $59-90$.

[30] M. Kočvara, A. Mielke and T. Roubíček, A rate-independent approach to the delamination problem. Math. Mech. Solids 11 (2006) 423-447.

[31] M. Kružík, A. Mielke and T. Roubíček, Modelling of microstructure and its evolution in shape-memory-alloy single-crystals, in particular in CuAlNi. Meccanica 40 (2005) 389-418.

[32] V.I. Levitas, The postulate of realizibility: formulation and applications to postbifurcational behavior and phase transitions in elastoplastic materials. Int. J. Eng. Sci. 33 (1995) 921-971.

[33] A. Mainik, A rate-independent model for phase transformations in shape-memory alloys. Ph.D. Thesis, Fachbereich Mathematik, Universität Stuttgart, Germany (2004).

[34] A. Mainik and A. Mielke, Existence results for energetic models for rate-independent systems. Calc. Var. 22 (2005) 73-99.

[35] A. Mainik and A. Mielke, Global existence for rate-independent gradient plasticity at finite strains. J. Nonlinear Science (2008) DOI: 10.1007/s00332-008-9033-y (published online).

[36] A. Mielke, Energetic formulation of multiplicative elasto-plasticity using dissipation distances. Cont. Mech. Thermodynamics 15 (2003) 351-382.

[37] A. Mielke, Evolution of rate-independent systems, in Handbook of Differential Equations: Evolutionary Equations, C. Dafermos and E. Feireisl Eds., Elsevier, Amsterdam (2005) 461-559. 
[38] A. Mielke and S. Müller, Lower semicontinuity and existence of minimizers for a functional in elastoplasticity. Z. Angew. Math. Mech. 86 (2006) 233-250.

[39] A. Mielke and T. Roubíček, Rate-independent model of inelastic behaviour of shape-memory alloys. Multiscale Model. Simul. 1 (2003) 571-597.

[40] A. Mielke and T. Roubíček, Rate-independent damage processes in nonlinear inelasticity. Math. Models Methods Appl. Sci. 16 (2006) 177-209.

[41] A. Mielke and F. Theil, A mathematical model for rate-independent phase transformations with hysteresis, in Models of Continuum Mech. in Anal. and Engineering, H.-D. Alber, R. Balean and R. Farwig Eds., Shaker Ver., Aachen (1999) $117-129$.

[42] A. Mielke and F. Theil, On rate-independent hysteresis models. Nonlin. Diff. Eq. Appl. 11 (2004) 151-189.

[43] A. Mielke and A. Timofte, An energetic material model for time-dependent ferroelectric behavior: existence and uniqueness. Math. Meth. Appl. Sci. 29 (2006) 1393-1410.

[44] A. Mielke, F. Theil and V.I. Levitas, A variational formulation of rate-independent phase transformations using an extremum principle. Arch. Ration. Mech. Anal. 162 (2002) 137-177.

[45] A. Mielke, T. Roubíček and U. Stefanelli, Relaxation and $\Gamma$-limits for rate-independent evolution equations. Calc. Var. P.D.E. 31 (2008) 387-416.

[46] A. Mielke, L. Paoli and A. Petrov, On the existence and approximation for a 3D model of thermally induced phase transformations in shape-memory alloys. SIAM J. Math. Anal. (submitted) (WIAS Preprint 1330).

[47] A. Mielke, T. Roubíček and J. Zeman, Complete damage in elastic and viscoelastic media and its energetics. Comput. Methods Appl. Mech. Engrg. (submitted) (WIAS preprint 1285).

[48] S. Müller, Variational models for microstructure and phase transitions, in Calculus of variations and geometric evolution problems, S. Hildebrandt et al. Eds., Lect. Notes in Math. 1713, Springer, Berlin (1999) 85-210.

[49] P. Pedregal, Parametrized Measures and Variational Principles. Birkhäuser, Basel (1997).

[50] P. Plecháč and T. Roubíček, Visco-elasto-plastic model for martensitic phase transformation in shape-memory alloys. Math. Meth. Appl. Sci. 25 (2002) 1281-1298.

[51] K.R. Rajagopal and T. Roubíček, On the effect of dissipation in shape-memory alloys. Nonlinear Anal. Real World Appl. 4 (2003) 581-597.

[52] H. Romanowski and J. Schröder, Modelling of the nonlinear ferroelectric hysteresis within a thermodynamically consistent framework, in Trends in Applications of Math. to Mech., Y. Wang and K. Hutter Eds., Shaker Ver., Aachen (2005) 419-428.

[53] T. Roubíček, A note on an interaction between penalization and discretization, in Proc. IFIP-IIASA Conf., Modelling and Inverse Problems of Control for Distributed Parameter Systems, A. Kurzhanski and I. Lasiecka Eds., Lect. Notes in Control and Inf. Sci. 154, Springer (1991) 145-150.

[54] T. Roubíček, Dissipative evolution of microstructure in shape memory alloys, in Lectures on Applied Mathematics, H.-J. Bungartz, R.H.W. Hoppe and C. Zenger Eds., Springer, Berlin (2000) 45-63.

[55] T. Roubíček and M. Kružík, Microstructure evolution model in micromagnetics. Z. Angew. Math. Phys. 55 (2004) $159-182$.

[56] T. Roubíček and M. Kružík, Mesoscopic model for ferromagnets with isotropic hardening. Z. Angew. Math. Phys. 56 (2005) $107-135$.

[57] T. Roubíček and M. Kružík, Mesoscopic model of microstructure evolution in shape memory alloys, its numerical analysis and computer implementation, in 3rd GAMM Seminar on microstructures, C. Miehe Ed., GAMM Mitteilungen 29 (2006) $192-214$.

[58] T. Roubíček, M. Kružík and J. Koutný, A mesoscopical model of shape-memory alloys. Proc. Estonian Acad. Sci. Phys. Math. 56 (2007) 146-154.

[59] P. Rybka and M. Luskin, Existence of energy minimizers for magnetostrictive materials. SIAM J. Math. Anal. 36 (2005) 2004-2019.

[60] Y. Shu, K. Bhattacharya, Domain patterns and macroscopic behaviour of ferroelectric materials. Phil. Mag. B 81 (2001) 2021-2054

[61] J.C. Simo, Numerical analysis and simulation of plasticity, in Handbook of Numer. Anal., P.G. Ciarlet and J.L. Lions Eds., Vol. VI, Elsevier, Amsterdam (1998) 183-499.

[62] J.C. Simo and J.R. Hughes, Computational Inelasticity. Springer, Berlin (1998).

[63] R. Temam, Mathematical problems in plasticity. Gauthier-Villars, Paris (1985).

[64] R. Tickle, Ferromagnetic shape memory materials. Ph.D. Thesis, University of Minnesota, Minneapolis, USA (2000).

[65] A. Visintin, Strong convergence results related to strict convexity. Comm. Partial Diff. Eq. 9 (1984) 439-466.

[66] A. Visintin, Modified Landau-Lifshitz equation for ferromagnetism. Physica B 233 (1997) 365-369.

[67] A. Visintin, Maxwell's equations with vector hysteresis. Arch. Ration. Mech. Anal. 175 (2005) 1-37.

[68] A. Vivet and C. Lexcellent, Micromechanical modelling for tension-compression pseudoelastic behaviour of AuCd single crystals. Eur. Phys. J. Appl. Phys. 4 (1998) 125-132. 